\title{
Metagenomics Revealing Molecular Profiling of Microbial Community Structure and Metabolic Capacity In Bamucuo, Tibet
}

\section{Cai Wei}

Shanghai Ocean University

Dan Sun

Shanghai Ocean University

Wenliang Yuan

Jiaxing University

Lei Li

Fudan University

Chaoxu Dai

Shanghai Ocean University

\section{Zuozhou Chen}

Shanghai Ocean University

Xiaomin Zeng

Central South University Xiangya Public Health School

Shihang Wang

Shanghai Ocean University

\section{Yifan Tang}

Hunan Normal University School of Medicine

\section{Shouwen Jiang}

Shanghai Ocean University

\section{Zhichao Wu}

Shanghai Ocean University

\section{Xiaoning Peng}

Hunan Normal University School of Medicine

\section{Linhua Jiang}

Fudan University

Sihua peng ( $\nabla$ shpeng@shou.edu.cn )

Shanghai Ocean University https://orcid.org/0000-0001-7231-666X 
Keywords: shotgun metagenomics, microbial community, extreme environment, Tibet, Qinghai-Tibet Plateau

Posted Date: May 24th, 2021

DOI: https://doi.org/10.21203/rs.3.rs-505014/v1

License: (c) (1) This work is licensed under a Creative Commons Attribution 4.0 International License. Read Full License 


\section{Abstract}

Background: The Qinghai-Tibet Plateau (QTP) is the highest plateau in the world, and the microorganisms there play vital ecological roles in the global biogeochemical cycle; however, detailed information on the microbial communities in QTP is still lacking.

Results: Here, we performed a landscape survey of the microorganisms in Bamucuo, Tibet, resulting in 160,212 (soil) and 135,994 (water) contigs by shotgun metagenomic methods, and generated 75 nearly complete metagenome-assembled genomes (MAGs). Proteobacteria, Actinobacteria and Firmicutes were found to be the three most dominant bacterial phyla, while Euryarchaeota was the most dominant archaeal phylum. Surprisingly, Pandoravirus salinus was found in the soil microbial community. The KEGG annotations showed that the genes related to metabolism accounted for $62.9 \%$ (soil) and $58.4 \%$ (water), respectively. Of the 75 MAGs, 63 were found to contain the genes related to all the six carbon fixation pathways, and the heavy metal resistance genes, the pressure response subsystem, and the secondary metabolite biosynthesis gene clusters (BGCs) were discovered to be abundant.

Conclusions: We concluded that the microorganisms in Bamucuo fix carbon mainly through the 3hydroxypropionic bi-cycle pathway; this study, for the first time, characterized the microbial community composition and metabolic capacity in QTP high-altitude areas (with an altitude of 4,555 meters), confirmed that QTP is a huge and valuable resource bank in which more new non-resistant antibiotics and many other bioactive substances could be developed, and provided the expanded information for further microbial community studies in QTP.

\section{Background}

The Qinghai-Tibet Plateau (QTP) is the highest plateau on the earth and is known as the "third Pole" of the world, which is one of the most important water resources in East Asia and plays a vital role in regulating global climate change [1,2]. Over the past 50 years, QTP has been warming at more than twice the global average [2], and the increase of temperature leads to the increase of soil carbon emissions. QTP is generally a carbon sink, but in the near future, warming may have a significant impact on the net ecosystem carbon balance of the ecosystem, which will gradually weaken the function of QTP carbon sink [3,4]. There are many microorganisms in QTP, which is one of the most important life forms in the extreme environments. Through various metabolic pathways, the microorganisms form the basis of global carbon, nitrogen and various nutrient cycles $[5,6]$, and affect the global climate change to a certain extent, especially to the emission of the greenhouse gases $\left(\mathrm{CO}_{2}, \mathrm{CH}_{4}, \mathrm{~N}_{2} \mathrm{O}\right)$ [7-9]. However, up to now, the diversity information and metabolic potential of the microbial communities in QTP are still lacking. To address this issue, we initiated this research by using metagenomics to increase the understanding of the interactions between the microorganisms and the environments.

With the development of sequencing technology, it is now possible to obtain information from unculturable microorganisms through metagenomics [10]. In recent years, the related studies have mainly 
focused on the permafrost and the Qaidam Basin, which is located at an altitude of 3,000 meters or below.

The permafrost in QTP is one of the most sensitive areas for climate warming. Due to its unique and fragile characteristics, the diversity of the microbial communities has become the focus of most research [11-14]. Han et al. revealed the structure and diversity of Keke Salt Lake microbial community [15]. Xing's research on the soil microbial community in Qaidam Basin showed that the environmental factors are the main driving force for the formation of bacterial community structure [16]. However, in QTP with an altitude of more than 4,000 meters, there are few environmental microbiology studies, which are mainly based on 16S Ribosomal RNA Gene Sequencing (16S) rather than shotgun metagenomic sequencing [11, 13-17]. Compared with shotgun metagenomic sequencing, $16 \mathrm{~S}$ has obvious deficiencies: 1) it cannot fully detect the abundance of the microbial community; 2 ) it can only assign microorganisms to the genus level, but not to specific species; and 3) it only sequence some fragments of the certain regions of the bacterial genome, and the whole microbial genome information cannot be obtained $[18,19]$.

Shotgun metagenomic sequencing improves the accuracy of species detection, not only providing a stronger and more reliable assessment of microbial diversity, but also providing valuable information on the metabolic potential of microbial communities [20-23]. The draft genomes reconstructed from the datasets can be used to understand the potential mechanisms of microbial adaptation to the extreme environments and their relationship with climate change $[24,25]$.

Bamucuo, not far from its sister lake Namcuo, is located in QTP, with an altitude of 4,555 meters. The lake is mainly fed by surface runoff surrounding. Here we used shotgun metagenomics to investigate the microbial community in Bamucuo. To the best of our knowledge, this study is the first shotgun metagenomics study of the environmental microbial community at an altitude of more than 4,000 meters in QTP.

\section{Materials And Methods}

The overall analysis process in this study is shown in Fig. 1.

\section{Sampling site and sample collection}

The soil and water samples involved in this study were collected at Bamucuo $\left(31^{\circ} 34^{\prime} \mathrm{N}, 90^{\circ} 6^{\prime} \mathrm{E}\right)$, at an altitude of 4,555 meters, Bango County, Nagqu Prefecture, Tibet, China, on June 20, 2017. We collected $30 \mathrm{~L}$ water sample at depth of $0.5 \mathrm{~m}$ through a filter membrane with a pore diameter of $0.22 \mu \mathrm{m}$, and the water temperature was $13^{\circ} \mathrm{C}$ when sampling. The ambient temperature was $19^{\circ} \mathrm{C}$. The soil sample was collected from $0.2 \mathrm{~m}$ below the surface, and then stored in ice boxes for bringing back to the laboratory. For simplicity, in this article, we refer to these two examples as s05-02 (soil) and s05-03 (water).

\section{DNA extraction and sequencing}


According to the manufacturer's instructions, genomic DNA was extracted using OMEGA's DNA extraction kit (http://www.omegabiotek.com.cn/, Guangzhou, China) and some modifications were made in the experimental procedure. The purity and concentration of the extracted genomic DNA were measured on NanoDrop 2000 spectrophotometer (Thermo Fisher Scientific, Massachusetts, United States), and the quality of the extracted genomic DNA was detected by $1 \%$ agarose gel electrophoresis. The total genomic DNA was stored in a $-20^{\circ} \mathrm{C}$ refrigerator. Then, the genomic DNA was sent to Shanghai Meiji Biomedical Technology Co., LTD for sequencing. The length of the library was $300 \mathrm{bp}$, and Hiseq 4000 highthroughput sequencer was used for sequencing.

\section{Metagenomic assembly and gene abundance analysis}

The raw reads were processed using fastp v. 0.19 .5 [26] for removing adapter sequences at the $3^{\prime}$ and $5^{\prime}$ terminal, and the reads with the length less than $50 \mathrm{bp}$ after shearing, the reads with an average quality value (Phred value) less than 20 , and the reads containing $\mathrm{N}$ bases were all removed. FastQC v. 0.11.9 [27] was used to perform the sequence quality controlling. The assembly module of MetaWRAP v. 1.2.1 [28] was then used for assembling, which include the assembly software MEGAHIT v 1.1.3 [29], setting the parameters as follows: minimum contig length of $1000 \mathrm{bp}$, and k-mer sizes of 21, 29, 39, 59, 79, 99, 119 and 141, respectively. BBMap v. 38.87 (https://sourceforge.net/projects/bbmap/) was used to map clean reads to each contig for calculating the coverage information. Prokka v. 1.14.6 [30] was used to annotate the assembled sequence, with all the parameters to be the default values. Salmon v. 1.3.0 [31] was then used to estimate the genetic abundance for each sample.

\section{Species classification and functional annotation}

We integrated the annotation results obtained by Kraken2 v. 2.1.1 [32] and Bracken v. 2.6.0 [33], viewing the results of the species annotation by Krona v. 2.7.1 [34].

Using Diamond v. 2.0.5 [35] as a aligner, and blastp v. 2.5.0+ [36] alignments (e-value $\leq 1 \mathrm{E}-5$ ) were performed against Non-Redundant Protein Sequence Database (NCBI-nr), Carbohydrate-Active Enzyme Database (CAZy) [37], Pathogen-Host Interactions Database (PHI) [38], Virulence Factors Database (VFDB) [39], and Biocide and Metal Resistance Genes Database (BacMet) [40], respectively. Rgi v. 5.1.1, based on Comprehensive Antibiotic Resistance Database (CARD) [41], was employed to predict the resistomes. The Kyoto Encyclopedia of Genes and Genomes (KEGG) [42] annotation was performed using KAAS online analysis website [43]. Clusters of Orthologous Groups of Proteins (COG) [44] and Gene Ontology (GO) [45-47] functional annotation were performed using emapper v. 2.0 .1 [48] based on the eggNOG orthology data [49], and then Web Gene Ontology Annotation Plot (WEGO v. 2.0) [50] was used to classify the GO functions.

\section{Metagenomic binning}

All the data of the two samples were binned to generate the draft genomes with the binning module in MetaWRAP [28], in which three modules (MetaBAT2 [51], MaxBin2 [52], and CONCOCT [53]) were selected to perform the binning operation separately to obtain three different binning results, setting the minimum 
contig length to be 1500. CheckM v 1.0.12 [54] was employed to calculate the completeness and contamination $[55,56]$. Then, bin_refinement module in MetaWRAP was used to obtain the bins with the completeness of more than $70 \%$ and the contamination of less than $10 \%$ (parameter: $-c 70-x 10$ ), and these bins were called metagenome-assembled genomes (MAGs).

By using the MetaWRAP pipeline [28], the clean reads of the each sample were mapped to the assembled MAGs by using the quant_bins module; the relative abundance of all the MAGs was estimated; a preliminary gene annotation for each MAG was obtained by using the annotate_bins module; the distribution of the contigs in each bin was visualized by the blobology module.

\section{Taxonomic classification and phylogenetic analysis}

GTDB-tk v. 1.3.0 [57] "classify" workflow was used to conduct species classification and to calculate the ANI values of the MAGs based on Genome Taxonomy Database (GTDB) [58, 59]. For phylogenetic analysis, we first searched the GTDB database to find the corresponding reference genomes which are closest to each MAG (Table S1), and then used GTDB-tk [57] "align" method to perform multiple sequence alignments of the marker genes in the MAGs and their reference genomes. After importing the above alignment results, the "infer" method (FastTree v. 2.1.10) [60] was used to construct a phylogenetic tree under the Whelan-And-Goldman (WAG) model, with the parameters to be set to "default". Finally, the annotation information was refined using ITOL v. 5.7 [61].

\section{Metabolism analysis}

The carbon degradation enzymes were identified using dbCAN [62]. We examined the KEGG [42] biochemical maps for the six known carbon fixation pathways (reductive acetyl-CoA, 3hydroxypropionate/4-hydroxybutylate cycle, 3-hydroxypropionate bi-cycle, dicarboxylate/4hydroxybutyrate cycle, reductive tricarboxylic acid cycle, and calvin cycle $[55,63])$ and identified the genes encoding the enzymes involved in methanogenesis and methane oxidation; the genes related to nitrogen and sulfur metabolism were investigated (Table S2). The percentage of the number of the annotated genes to the total number of the genes is used to characterize the completeness of the pathway.

\section{Microbial stress response and heavy metal resistance analyses}

Diamond [35] was used to perform the alignments for all the MAGs (e-value < 1E-5) against BacMet Database [40]. Then, the 75 MAGs were submitted to Rapid Annotation using Subsystem Technology (RAST v. 2.0) [64] to obtain the functional annotations of the SEED subsystem [65, 66].

\section{BGCs and ARGs identification}

The MAGs were submitted to the Anti-SMASH v. $5.2 .0[67,68]$ web version to screen the secondary metabolite biosynthetic gene clusters (BGCs) of these draft genomes, with the model set to 'strict'. Rgi [41] was used to predict the resistomes for the MAGs. 


\section{Result}

\section{Quality controlling and metagenome assembling}

Shotgun sequencing produced $29,623,109,936 \mathrm{bp}$ and $24,284,563,524 \mathrm{bp}$ of unassembled raw bases from the soil (s05-02) and water (s05-03) samples (Table 1), respectively. In total, 194,202,138 (98.99\%) and $159,527,496(99.19 \%)$ reads passed the quality control in the soil and water sample dataset, respectively (Table 1$)$.

After assembling, a total of 160,212 (soil) and 135,994 (water) contigs were generated (Table 1). For the two samples, the longest contigs were 833,193 bp and 286,311 bp, GC content were $60.18 \%$ and $51.33 \%$, and N50 length were 2,289 bp and 3,841 bp, respectively.

After Prokka [30] annotation, 351,242 genes were obtained from the soil sample and 390,943 genes from the water sample (Table 2).

\section{Metagenome-assembled genomes (MAGs)}

The CheckM [54] analysis showed that 75 MAGs were reconstructed with the completeness $>70 \%$ and contamination $<10 \%$, including 18 from the soil sample and 57 from the water sample (Fig. 2a and Fig. 2b). A total of 20 MAGs met the high-quality standard with the completeness $>95 \%$ and contamination $<5 \%$, accounting for $26.67 \%$ of all the MAGs. Table S3 lists the statistics of the 75 bins including the completeness, contamination, GC value, N50, genomic size, and GTDB $[58,59]$ species classification. The original reconstructed MAGs have been reassembled by using reassemble_bins module in MetaWRAP [28] and their genomic integrities have been significantly improved (Fig. 2c and Fig. 2d).

\section{Microbial community composition analysis}

The microbial community composition of the soil sample was visualized by Krona [34] (Fig. 3). The results showed that bacteria accounting for $99.5 \%$ were dominant, with archaea accounting for $0.3 \%$ and viruses accounting for $0.08 \%$. The three most abundant phyla in bacteria were Proteobacteria $(54.0 \%)$, Actinobacteria (18.6\%) and Firmicutes (9.8\%), and another 29 phyla were identified (Table S4a), such as Bacteroidetes (8.9\%), Gemmatimonadetes (3.3\%), Planctomycetes (1.3\%), and Acidobacteria (1.1\%). For the most abundant phylum, Proteobacteria, 112 families (Table S4b) were identified, in which Sphingomonadaceae (34.9\%) was the most abundant, followed by Bradyrhizobiaceae (7.1\%), Methylobacteriaceae (6.3\%), etc. At the genus level of the Proteobacteria, 408 genera were identified (Table S4c), in which Sphingomonas (22.9\%) had the highest abundance, followed by Sphingobium (4.6\%), Methylobacterium (4.4\%), etc. Among the Actinobacteria (Table S4d), we identified 46 families, in which Streptomycetaceae (22.7\%) was the most abundant, and at the genus level of the Actinobacteria, 141 genera were identified, in which Streptomyces (21.5\%), Conexibacter (7.5\%) and Micromonospora $(5.6 \%)$ were the three most abundant genera (Table S4e). A total of 2,400 bacterial species were identified 
(Table S5), including Bacillus cereus (5.1\%), Hymenobacter sedentarius (4.5\%), Gemmatirosa kalamazoonesis (3.0\%), Sphingomonas indica (2.3\%), Lactococcus piscium (1.6\%), etc.

In the soil sample, Euryarchaeota (81.9\%) accounted for the largest proportion in the archaea (Table S6a), and Thaumarchaeota (14.7\%) was the second most dominant phylum, followed by Crenarchaeota (1.7\%). Table S6b, c, $d, e$, and $f$ list the detailed information for the archaea at the class, order, family, genus, and species level, respectively.

Eighteen species of viruses were identified in the soil sample (Table S7). Surprisingly, Pandoravirus salinus (15.3\%), a giant virus, was the most abundant, followed by Bacillus virus 250 (11.5\%), Mycobacterium phage Jobu08 (7.7\%), etc.

In the water sample (Fig. 4), the bacteria accounted for the majority (99.1\%). Only a small portion was archaea (0.4\%), and the rest was viruses (0.5\%). At the phylum level, Proteobacteria ( $54.4 \%)$, Bacteroidetes (17.9\%) and Actinobacteria (15.1\%) were the most abundant phyla, and another 30 phyla were identified (Table S8a). Among the Proteobacteria, 124 families (Table S8b) were identified, including three most abundant families: Rhodobacteraceae (42.6\%), Comamonadaceae (14.8\%) and Pseudomonadaceae (3.8\%). Among the 464 genera identified in the Proteobacteria (Table S8c), Yoonia (22.4\%) was the most abundant, followed by Limnohabitans (6.8\%) and Pseudomonas (3.7\%). For the Actinobacteria, 46 families (Table S8d) were identified, of which Microbacteriaceae (23.3\%) was the most abundant, and 150 genera (Table S8e) were identified, of which Streptomyces (13.9\%), Corynebacterium (5.6\%) and Pontimonas (5.1\%) accounted for the largest proportion. A total of 2,714 bacterial species (Table S9) were identified, in which Yoonia vestfoldensis (12.2\%), Belliella baltica (5.3\%), Limnohabitans sp. 63ED37-2 (3.6\%), Aquiflexum balticum (1.8\%) and Cyanobium sp. NIES-981 (1.2\%) were dominant.

Among the archaea in the water sample, Euryarchaeota (98.8\%) accounted for the largest proportion (Table S10). Table S10b, c, d, e, and f list the detailed information for the archaea at the class, order, family, genus, and species level, respectively.

In the water sample, a total of 52 species of viruses were identified (Table S11), in which the most abundant was Chrysochromulina ericina virus (12.7\%), followed by Phaeocystis globosa virus (8.8\%) and Synechococcus phage S-PM2 (5.9\%).

\section{Phylogenetic analysis}

A phylogenetic analysis for the 75 MAGs was performed. Initially, using CheckM [54] to classify the MAGs, 38 of the 75 MAGs (50.67\% of the datasets) were not assigned to a specific taxonomic name below a domain. Subsequently, we further refined the classification of all the MAGs by using GTDB-tk [57], and bin 5 from the soil sample with the $98.06 \%$ average nucleotide identities (ANIs) was even successfully classified to the species level. Of the 75 MAGs, 28 (37.33\%) lacked a genus-level match, and only one (bin13 from the water sample) lacked a family-level match (Table S3). 
The phylogenetic tree constructed with the MAGs (Fig. 5) showed that the 75 MAGs could be taxonomically assigned to 10 phyla, including main members: Proteobacteria (16 MAGs, five from the soil sample and 11 from the water sample), Bacteroidota (22 MAGs, two from the soil sample and the remaining 20 from the water sample), Actinobacteriota (17 MAGs, three from the soil sample and 14 from the water sample), etc. The microbial community composition of the soil and water sample was roughly similar, which may be due to the fact that sampling points of the two samples were very close. However, at the phylum level and below, the species composition of the two samples gradually showed differences.

\section{Metagenomic functional annotation}

For the two samples, a total of 232,679 and 294,919 genes were annotated against nr database, 104,704 and 117,467 genes against KEGG [42] database, and 45,638 and 59,921 genes against GO [46] database, respectively. More annotation results are shown in Table 2.

The COG [44] functional annotation results were visualized using ggplot2 (Fig. 6a). Except for function unknown (12.8\%), the three most abundant categories in the soil sample were replication, recombination and repair (4.7\%), translation, ribosomal structure and biogenesis (3.9\%), and transcription (3.9\%). In the water sample, except for function unknown (11.0\%), translation, ribosomal structure and biogenesis $(4.3 \%)$ was the most abundant, followed by replication, recombination and repair (3.6\%), amino acid transport and metabolism (3.5\%), etc.

WEGO [50] was used to show the three main categories (cellular component, molecular function and biological process) and the first two levels of the GO terms, with bar charts showing the number of the genes annotated. In the soil sample (Fig. 6b), the dominant category was biological process (35.5\%), followed by molecular function (33.0\%) and cellular component $(31.5 \%)$. We found that among the biological processes, the top three $\mathrm{GO}$ terms were cellular process $(33,656)$, metabolic process $(32,600)$ and response to stimulus (8,678); among the molecular functions, the top three were catalytic activity $(27,005)$, binding $(17,692)$ and structural molecule activity $(3,089)$; and among the cellular components, the top three were cell $(33,716)$, cell part $(33,716)$ and membrane $(14,342)$. Whereas in the water sample (Fig. 6b), biological process (35.4\%) was the dominant category, followed by molecular function (32.7\%) and cellular component (31.9\%). The top three $\mathrm{GO}$ terms were as follows: cellular process $(45,113)$, metabolic process $(43,200)$ and response to stimulus $(12,611)$ among the biological processes; catalytic activity $(35,860)$, binding $(24,853)$ and structural molecule activity $(4,247)$ among the molecular functions; and cell $(45,101)$, cell part $(45,101)$ and membrane $(19,524)$ among the cellular components.

ggplot2 was used to display the annotation information at the first two levels of KEGG [42], with the bar graphs representing the number of the annotated genes for each metabolic pathway. For the soil sample (Fig. 6c), the genes related to metabolism (about $62.9 \%$ of the datasets) were the most abundant, and the rest were genetic information processing (9.7\%), environmental information processing $(9.4 \%)$, human diseases $(7.3 \%)$, cellular processes $(6.8 \%)$, and organismal systems (3.9\%). Further analysis on the metabolism showed that the most abundant was carbohydrate metabolism (23.6\%), followed by amino 
acid metabolism (20.5\%), metabolism of cofactors and vitamins (10.7\%), energy metabolism (10.1\%), etc.

Similarly, for the water sample (Fig. 6c), the most abundant metabolic function module was metabolism $(58.4 \%)$, followed by human diseases $(9.7 \%)$, environmental information processing $(8.5 \%)$, cellular processes $(6.4 \%)$, organismal systems $(6.3 \%)$, and genetic information processing (6.2\%). In metabolism, carbohydrate metabolism (24.5\%) was the most abundant, followed by amino acid metabolism (20.7\%), metabolism of cofactors and vitamins (10.6\%), energy metabolism (9.7\%), etc.

\section{Metabolic potential analyses based on the MAGs}

Carbon degradation: The 75 MAGs contained abundant hydrolases from the 101 glycoside hydrolases (GH) family (Table S12), among which the MAGs containing GH13 accounted for $77.3 \%$ (58 out of 75), and the MAGs containing GH3 and GH23 accounted for 76.0\% (57 out of 75), respectively. The enzymes of interest included alpha-amylase, beta-amylase, glucoamylase, alpha-glucosidase, isoamylase and pullulanase (involved in starch degradation); xylanase, beta-mannanase, alpha-L-arabinofuranosidase, alpha-D-glucuronidase and beta-xylosidase (involved in hemicellulose degradation); endo-1,4-glucanase, exo- $\beta-1,4$-glucanases and $\beta-1,4$-glucosidases (involved in cellulose degradation); and other carbohydrate degradation enzymes.

Carbon fixation: Carbon fixation capacity was examined for each of the 75 MAGs (Fig. 7). Almost all the MAGs had the genes encoding the enzymes involved in the 3-hydroxypropionate bi-cycle, reductive tricarboxylic acid cycle, and calvin cycle pathways (related enzymes are listed in Table S2). The two MAGs (bin2 and bin 18 in the soil sample) possessed more than $50 \%$ complete 3 -hydroxypropionate bicycle pathway. In the results, 27 MAGs (not listed) had one or more genes encoding the key enzymes, 2oxoglutarate: ferredoxin oxidoreductase, pyruvate: ferredoxin oxidoreductase, and ATP-citrate lyase, of the reductive tricarboxylic acid cycle pathway. Although the calvin cycle pathway was relatively complete based on the KEGG modules, the key enzyme, ribulose-1,5-bisphosphate carboxylase-oxygenase (RuBisCO), was only identified in the two MAGs (bin9 in the soil sample and bin32 in the water sample), and another key enzyme of this pathway, phosphoribulokinase (PRK), was identified in the two MAGs (bin15 and bin44 in the water sample). Further, except for the two MAGs (bin9 in the soil sample and bin15 in the water sample), other MAGs had the genes encoding the enzymes involved in the reductive acetyl-CoA pathway, and most of the MAGs had the key enzyme, acetyl-CoA synthase, of this pathway. Except for bin 12 in the water sample, 74 MAGs had the genes related to the dicarboxylate/4hydroxybutyrate cycle pathway; the genes encoding the enzymes that related to the 3hydroxypropionate/4-hydroxybutylate cycle pathway existed in 64 MAGs.

Methane metabolism: As we know, microbial methanogenesis and methane oxidation play essential roles in the biogeochemical cycles of the earth system. We found that, except bin54 in the water sample, almost all the MAGs contained genes related to the methanogenesis pathway, however, this pathway was incomplete. It can be seen from Fig. 7 that bin42 in the water sample possessed 17\% complete methanogenesis pathway. The key enzyme of the methanogenesis pathway, methyl coenzyme M 
reductase (MCR), was found in 17 MAGs (not listed). Furthermore, no enzyme related to the methane oxidation pathway was identified in all the MAGs in the two samples (Fig. 7).

Nitrogen metabolism: As shown in Fig. 7, the genes related to the assimilation nitrate reduction pathway were identified in eight MAGs, while 13 MAGs had the genes encoding the enzymes related to the dissimilation nitrate reduction pathway. Only one MAG had the gene encoding the key enzyme, respiratory nitrate reductase, of the dissimilation nitrate reduction pathway. Besides, the genes related to the nitrogen fixation pathway were identified in three MAGs (Fig. 7), while the nitrogenase genes (nif, vnf and anf) were not found. Four MAGs (Fig. 7) had the genes related to the denitrification pathway, while no MAG had the genes related to the nitrification pathway. There were only two MAGs which had the genes encoding the key enzyme, nitrite reductase, of the denitrification pathway.

Sulfur metabolism: For sulfur metabolism, 52 MAGs (Fig. 7) had the genes related to the assimilatory sulfate reduction pathway, while eight MAGs had the genes related to the dissimilatory sulfate reduction pathway and 12 MAGs had the genes related to the thiosulfate oxidation by SOX complex pathway. Among the 12 MAGs, the marker gene, soxB, used to analyze the thiosulfate oxidation by SOX complex pathway was found in the ten MAGs (bin1 in the soil sample and bin1, bin2, bin35, bin41, bin44, bin46, bin51, bin54, bin55 in the water sample).

\section{Stress response and metal resistance analyses}

We investigated the subsystems of all the MAGs, with the results showing that more than 19 types of the subsystem [65] categories were identified, including the three most abundant categories: carbohydrates, amino acids and derivatives, and protein metabolism (see Table S13 for more information). All the MAGs contained respiration, stress response, and virulence, disease and defense categories. However, there were only four MAGs (one Planctomycetota MAG and three Proteobacteria MAGs) containing photosynthesis category. All RAST [64] annotations showed that the highest subsystem coverage was $33.0 \%$, and the lowest was $12.0 \%$.

Both the samples contained many stress response subsystems (Table S14), of which the detoxification subsystems were found in all the MAGs, the oxidative stress subsystems were found in the abundant MAGs except for the two Actinobacteriota MAGs, and the osmotic stress subsystems were found in 54 MAGs. Besides, we found that two MAGs (one Bacteroidota MAG and one Proteobacteria MAG) in the water sample had proteins associated with cold shock (CspA family of proteins). These proteins are induced when the temperature drops, allowing cells to adapt to lower temperature [69].

The annotation for heavy metal resistance genes revealed diverse resistance genes that confer resistance to 19 heavy metals such as silver, copper, iron, nickel, zinc, and antimony, including $\operatorname{act} P, \operatorname{cop} A, \operatorname{cop} B$, silP, acn, $\operatorname{ctp} C$, ziaA, etc (related genes are listed in the Table S15). The results showed that more than 1,064 heavy metal resistance genes were identified in all the MAGs (Fig. 8), among which the heavy metal resistance genes conferring resistance to multi-metals were abundant. Furthermore, the genes conferring resistance to antimony, arsenic, molybdenum and tungsten have been identified in all MAGs. 


\section{Biosynthetic gene cluster (BGC) identification}

By using Anti-SMASH [67], a total of 270 putative BGCs clusters of secondary metabolites were identified in the 75 MAGs, revealing that the two samples contained 25 types of BGCs such as terpene, T3PKS, NRPS, lanthipeptide, and bacteriocin (Table S16); there were 96 terpenes (35.56\%) categories, 40 NRPS (14.81\%) categories, 35 T3PKS (12.97\%) categories, and 27 bacteriocin $(10.00 \%)$ categories. We found that most NRPSs existed in the Acidobacteriota MAGs, for example, 25 of 40 NRPSs existed in the Acidobacteriota MAGs. Furthermore, terpenes were found in 61 of all the MAGs. Similarly, T3PKSs were found in many MAGs in the two samples, NRPSs were found in the Acidobacteriota MAGs and the Bacteroidota MAGs, and PKSs were found in the Bacteroidota MAGs and the Actinobacteria MAGs. Eleven bacteriocins were found in the Cyanobacteria MAG. Interestingly, some of the BGCs identified in this study were highly similar to known gene clusters, while the others were not closely related to any known gene clusters.

\section{Antibiotics resistance gene (ARG) annotation}

ARGs were found in 15 of the 75 MAGs, including adeF, rpsL (mycobacterium tuberculosis rpsL mutations conferring resistance to streptomycin), etc. Among these $15 \mathrm{MAGs}$, adeF existed in all the soil sample MAGs, and ampS existed in bin10. In the water sample, besides adeF and ampS, rpsL accounted for the majority (5 out of 8). The detailed ARGs annotation information is shown in Fig. 9.

\section{Discussion}

\section{Microbial community structure}

Extreme environment is an ideal model to illustrate the adaptability of microorganisms [70]. To the best of our knowledge, our study, for the first time, reveals the composition of microbial communities in the surface water and nearshore soil in Bamucuo, Tibet. We found that the dominant bacterial phyla of the soil and water microbial communities were Proteobacteria, Actinobacteria and Firmicutes (Fig. 3 and Fig. 4), which were also found to be dominant phyla in other areas in QTP such as Qaidam Basin [16] and Qinghai Lake [71]. As the dominant bacteria we found in this study, Proteobacteria plays an essential role in various energy metabolism in QTP [16, 72], and at the class level of the Proteobacteria, Alphaproteobacteria was the most prevalent in Bamucuo microbial community, with the most common genus belonging to Sphingomonas (22.4\%) in the soil microbial community (Table S4c) and Yoonia (22.9\%) in the water microbial community (Table S8c); however, a study on Lake Meyghan reported that Gammaproteobacteria is the most abundant class of Proteobacteria [73]. We believed that this is a unique feature of the microbial composition in Bamucuo because of the differences in environmental conditions (such as altitude). Actinobacteria is the main source of new antibiotics and bioactive molecular candidates [74]. However, many of the existing antibiotics used by human have developed drug resistance [75]. Therefore, the discovery of many Actinobacteria in the water and soil microbial communities in Bamucuo may become important natural resources to develop new antibiotics. 
Firmicutes, one of the abundant phyla in Bamucuo, can also be found in desert [76] and deep sea sediment [77] due to their spores, so our study confirmed that Firmicutes could adapt to various extreme environments including QTP.

Furthermore, we confirmed that Euryarchaeota is the most representative archaea in the Bamucuo microbial community in QTP, and similar observation in Lonar soda Lake in India was reported by Chakraborty et al. [78], suggesting that the phylum Euryarchaeota can adapt to the most extreme environmental conditions. However, Thaumarchaeota and Crenarchaeota were only identified in the soil sample in Bamucuo, suggesting Thaumarchaeota and Crenarchaeota might be more suitable for survival in the soil.

Philippe et al. reported that Pandoravirus, the largest known virus to date, was isolated from the sediments of the estuary of central Chile and the freshwater pond near Melbourne (Australia) [79]. However, in our research, Pandoravirus salinus was found in the nearshore soil, and this finding proved that the habitat of Pandoravirus may not only be in water.

In phylogenetic analysis (Fig. 5), we observed that in the same habitat, the species diversity of water microbial community is greater than that of soil microbial community. In summary, as a special extreme environment, the microbial community in Bamucuo has different characteristics from other extreme environments such as deep sea and desert.

\section{Carbon metabolism}

Microorganisms play very important roles in carbon fixation [80]. We found that the majority of the microbial community possessed relatively complete carbon fixation pathways (Fig. 7), suggesting that the microbial community in Bamucuo obtains energy through carbon fixation by autotrophic bacteria. Although many different carbon fixation pathways were found in both the samples, the 3hydroxypropionate bi-cycle (9.5\%-52.4\%), calvin cycle (11.8\%-52.9\%) and reductive tricarboxylic acid (2.3\%-37.2\%) pathways were the most complete (Fig. 7). Among them, the most represented carbon fixation pathway in the 75 MAGs was the 3-hydroxypropionate bi-cycle pathway, which is generally rarely found in most environments because of its high energy cost [81]. Interestingly, the abundant genes related to the 3-hydroxypropionate bi-cycle pathway were found in all the MAGs, and the key enzymes, acetyl-CoA carboxylase and propionyl-CoA carboxylase, of this pathway were present in most MAGs, suggesting that the 3-hydroxypropionate bi-cycle pathway may be dominant under extreme environmental conditions, such as low oxygen, low pressure, low temperature and high salinity. The calvin cycle pathway is the main $\mathrm{CO}_{2}$ fixation pathway, which is proved to be widely present in green plants and many autotrophic bacteria in most environments, such as trench [82]. However, even if the calvin cycle pathway was high complete, its key enzymes were lacking in our results. Compared with the calvin cycle pathway, the reductive tricarboxylic acid pathway has lower energy consumption, which was found in certain autotrophic mesophilic bacteria and archaea [81]. Our results showed that 27 MAGs had the key enzymes in the reductive tricarboxylic acid pathway, indicating that the microorganisms in Bamucuo may prefer to use this pathway instead of the calvin cycle pathway. In addition to the most 
common three carbon fixation pathways mentioned above, the three less common pathways were the reductive acetyl-CoA (0-30.0\%), dicarboxylate/4-hydroxybutyrate (0-39.1\%), and 3-hydroxypropionate/4hydroxybutylate cycle (0-27.8\%) pathways. The reductive acetyl-CoA pathway, which requires strict anoxic conditions, plays a role in both psychrophiles and hyperthermophiles [81]. At least 73 MAGs had the key enzyme, acetyl-CoA synthase, of the reductive acetyl-CoA pathway (Fig. 7), but the completeness of this pathway is low. The reductive acetyl-CoA pathway was found to be the most abundant carbon fixation pathway in deep biosphere $[55,83]$, however, our results suggested that the microorganisms in Bamucuo may not use this pathway. The 3-hydroxypropionate/4-hydroxybutyrate cycle and the dicarboxylate/4hydroxybutyrate cycle pathways existed in thermophilic archaea, and the 3-hydroxypropionate/4hydroxybutylate cycle seems to be the dominant pathway in anoxic marine zones [84], which is different from our results.

Therefore, the 3-hydroxypropionate bi-cycle pathway is the preferred methods for microorganisms to fix inorganic carbon into organic carbon in Bamucuo.

\section{Methane metabolism}

In methane metabolism, $\mathrm{CO}_{2}$ is converted to $\mathrm{CH}_{4} \cdot \mathrm{CH}_{4}$ is the second largest greenhouse gas, accounting for about $17 \%$ of global warming impact [85], while $\mathrm{CO}_{2}$ accounting for about $60 \%$ [86]. The results showed that the most representative features of our samples are the low completeness of the methanogenesis pathway and the unpredicted methane oxidation pathway (Fig. 7). In the methanogenesis pathway of microbial communities, the methane was generated through the key enzyme, methyl-coenzyme M reductase (MCR), which was identified both in the KEGG [42] annotation results of the MAGs and the metagenomic data, whereas the study on Pangong Lake reported the absence of $M C R$ [87]. In the contigs of the two samples, the existence of methanogens, such as Methanobacterium, Methanosarcina and Methanocaldococcus, suggested that methanogens can survive in the hypoxic environment in Bamucuo and are crucial in the methane production.

Methane oxidation is an important process to slow down $\mathrm{CH}_{4}$ emission [88], which is performed by $\mathrm{CH}_{4}$ oxidizing bacteria (methanotrophs). We found that methanotrophs, such as Methylomonas,

Methylomicrobium, and Methylococcus, existed in the two samples. However, in the KEGG [42] annotation results, the key functional genes for methane oxidation were not found, which may be due to insufficient sequencing depth.

\section{Nitrogen cycle}

Because the MAGs possessed the incomplete nitrogen metabolism pathways, the nitrogen metabolism might rarely occur in energy metabolism in Bamucuo, Tibet. Nitrogen loss in QTP is mainly related to denitification [89]. In our study, there are few genes related to the denitrification pathway. However, denitrifying bacteria, such as Pseudomonas and Bacillus, were identified in the metagenomic data of both the samples, indicating that denitrification with nitrate as the terminal electron acceptor is a rare but indispensable pathway to remove nitrogen in Bamucuo. Nitrification is a key process in biogeochemical 
nitrogen cycling, which oxidizes ammonia to nitrate via nitrite [90]. In our result, nitrosifyer (such as Nitrosomonas and Nitrosococcus) and nitrifying bacteria (such as Nitrobacter and Nitrpspina) were low abundant, and the nitrification pathway was not identified, suggesting that nitrification in the surface water and soil microbial communities was relatively rare. However, Rasigraf et al. reported that nitrification and denitrification are the main processes in the Bothnian Sea sediment [91], which is contradictory to our results. It was previously reported that Proteobacteria plays an important role in the nitrogen cycle [92], and our results also confirm that the nitrogen fixation pathway has only been found in the Proteobacteria MAGs. In addition, the assimilatory nitrate reduction pathway and the dissimilatory nitrate reduction pathway were more abundant than other pathways of nitrogen cycle, indicating that these two pathways are relatively common in the microbial communities in Bamucuo, which is similar to the results reported by Rathour et al. [87].

\section{Sulfur cycle}

The microbial sulfur cycle has been proposed as the driving force for bacterial survival [93]. The sulfurreducing bacteria (e.g., Desulfobacterales, Desulfovibrionales, Desulfotomaculum and

Desulfosporosinus) in Bamucuo, which can perform anaerobic respiration utilizing sulfate $\left(\mathrm{SO}_{4}{ }^{2-}\right)$ as the terminal electron acceptor for reducing sulfate to hydrogen sulfide $\left(\mathrm{H}_{2} \mathrm{~S}\right)$, were found in the two samples; similar results in Pangong lake were reported by Rathour et al. [87]. The assimilative sulfate reduction pathway was originally reported in plants, but it has been shown to exist in certain bacteria, such as Allochromatium vinosum [94]. Our results showed that the assimilatory sulfate reduction pathway was relatively complete (Fig. 7) in Bamucuo, while the key functional gene for dissimilatory sulfate reduction, dissimilatory sulfite reductase, had not been identified, which indicated the lack of microorganisms that perform the functions of dissimilatory sulfate reduction.

The sulfur-oxidizing bacteria such as Sulfuritalea and Thiobacillus were present with a low abundance, and few genes related to the thiosulfate oxidation by SOX complex pathway were identified (Fig. 7). However, the key gene, $\operatorname{sox} B$, related to the thiosulfate oxidation by SOX complex pathway was found in most of the MAGs which possessed this pathway, so we believed that sulfur oxidation is important in the energy metabolism of the microorganisms in Bamucuo.

\section{Stress response and metal resistance}

The subsystem annotation showed that the abundant presence of certain stress response subsystems, such as osmotic stress, oxidative stress, and cold shock (Table S14), could help the microorganisms to withstand various stress conditions in QTP such as temperature, salinity, hypoxia, and low pressure. Koo et al. reported that almost the entire cold-shock family of proteins exists in Lake Untersee in Antarctica [95], however, the cold shock pathway was rarely found in our study, it may be due to the higher temperature during sampling.

The microbial community is the main driving force for metal resistance gene distribution, and a previous study showed that these genes are found mainly in the environments contaminated with heavy metals 
[96]. However, in our metagenomic data, abundant heavy metal resistance genes were identified (Fig. 8). A study supported that the pollution of heavy metals in QTP has been on the rise in recent years [97]. In addition, rainwater and runoff may cause microorganisms to accumulate in the lake, being more conducive to the enrichment of heavy metal resistance genes in the water and nearshore soil microbial communities.

A previous study on Yellow River have reported that heavy metals can induce DNA damage and oxidative stress [98]. Our study showed that the DNA repair genes (not listed), such as $\operatorname{rec} B$, $\operatorname{rec} C$, recD, and recF, were found in most of the MAGs, which is obviously the result of the microbial community in Bamucuo adapting to the environment with the abundant heavy metals.

\section{BGCs and ARGs}

Among the secondary metabolites of microorganisms, special attention is always paid to antibiotics. In recent years, some pathogens have developed "drug resistance" to certain antibiotic drugs, and the number of new antibiotics has steadily decreased [75]. Therefore, there is a urgent need to find new resources to obtain new antibiotic drugs. Obviously, QTP is the appropriate candidate because of less human activity. Many BGCs were identified in both the samples (Table S16), suggesting that there may be many novel natural product biosynthetic gene clusters in Bamucuo.

Terpenes are the largest and the most diverse natural compounds, and are widely distributed in the genomes of many plants, fungi and bacteria [99]. In our study, the content of terpenes was the highest (35.56\%) (Table S16), indicating that terpenes have the potential to become a new source to develop natural products, however, until now, little is known about their ecological functions in bacteria. Nonribosomal peptide synthases (NRPSs) and polyketide synthases (PKSs) that we pay more attention to are two families of modular mega-synthases [100], and NRPSs (14.81\%) and PKSs (15.93\%) were the most abundant types of BGCs except for terpenes (Table S16), which produce compounds such as antibiotics, antifungals, immunosuppressants, and iron-chelating molecules [101]. Many NRPS and PKS gene clusters were found in the Actinobacteria MAGs, Acidobacteria MAGs and Bacteroidetes MAGs, suggesting that besides Actinobacteria, Acidobacteria and Bacteroidetes may also be the potential natural product biosynthetic producers in Bamucuo. In summary, QTP can be a valuable resource bank for discovering new secondary metabolite clusters.

Antibiotic resistance gene is a new pollutant with mobility [102]. Our results showed that few ARGs were identified (Fig. 9) in the soil and water microbial communities in Bamucuo. However, studies in other regions reported that abundant ARGs have been found $[78,103]$. Therefore, we inferred that Bamucuo has not yet been extensively contaminated by the ARGs. Our study is of great significance in developing new antibiotics without resistance. Interestingly, rpsL was only identified in the Actinobacteriata MAGs (Fig. 9), indicating that Actinobacteriata may be the source of rpsL in Bamucuo. Related research also supports that some ARGs found in pathogenic bacteria come from Actinobacteriata that produce antibiotics [104].

\section{Conclusion}


In this study, we investigated the microbial ecosystem of Bamucuo in QTP. The results showed that the water and soil in Bamucuo have unique microbial community structure and metabolic process. The presence of Pandoravirus salinus in the soil provides important information for further exploring this unique microorganism and discovering more new giant virus. We concluded that the 3-hydroxypropionate bi-cycle pathway is the most representative carbon fixation pathway in Bamucuo; the contribution of the microorganisms in Bamucuo to the nitrogen cycle, sulfur cycle and methane cycle is very small. Since Actinobacteria was one of the dominant bacteria, QTP can become a valuable resource bank for developing new and non-resistant antibiotic drugs and other bioactive substances. This study provides many draft genomes and the genome annotation information for further in-depth study of the microbial ecology in QTP. Future studies are needed to combine microbial metabolomics data and geochemical data to address the relationship between environmental factors and microbial metabolism, which will help us to clarify the impact of microbial ecological change in QTP on global warming.

\section{Declarations}

\section{Acknowledgements}

Not applicable

\section{Authors' contributions}

S.H.P., X.N.P., and L.H.J. conceived of the study. C.W., D.S., L.L., and W.L.Y. participated in the study design. C.W., D.S., C.X.D., and S.H.W performed the wet-lab experiments. C.W., D.S., W.L.Y., Z.Z.C and Y.F.T. processed the raw data and performed the computational analyses. S.W.J. and Z.C.W designed the sampling scheme and guided the process of DNA extraction and metagenome sequencing. S.H.P and X.M.Z. guided the pipelines of all the analyses. C.W. wrote the first draft of the manuscript. S.H.P., X.N.P., and L.H.J. reviewed and revised the manuscript. All authors reviewed and approved the final version of the manuscript.

\section{Funding}

This research was supported by the Shanghai Natural Science Foundation (SNSF, grant no. 15zr1420800) to Sihua Peng, and partly supported by the National Science Foundation (NSF, grant no. 61775139) to Linhua Jiang and the National Science Foundation (NSF, grant no. 81472860) to Xiaoning Peng.

\section{Raw data availability}


All the raw sequencing data were deposited in the NCBI Sequence Read Archive (SRA) under accession numbers SAMN17838283 (soil sample-05-02) and SAMN17838284 (water sample-05-03). The metagenomic sequence data is bundled under NCBI BioProject number PRJNA700111.

\section{Ethics approval and consent to participate}

Not applicable

\section{Consent for publication}

Not applicable

\section{Competing interests}

The authors declare that they have no competing interests.

\section{References}

1. Chen B, Zhang X, Tao J, Wu J, Wang J, Shi P, et al. The impact of climate change and anthropogenic activities on alpine grassland over the Qinghai-Tibet Plateau. Agric For Meteorol. 2014;189-190:118. https://doi.org/10.1016/j.agrformet.2014.01.002.

2. Kuang $X$, Jiao JJ. Review on climate change on the Tibetan Plateau during the last half century. Journal of Geophysical Research: Atmospheres. 2016;121(8):3979-4007. https://doi.org/https://doi.org/10.1002/2015JD024728.

3. Piao SL, Tan K, Nan HJ, Ciais P, Fang JY, Wang T, et al. Impacts of climate and CO2 changes on the vegetation growth and carbon balance of Qinghai-Tibetan grasslands over the past five decades. Global Planet Change. 2012;98-99:73-80. https://doi.org/10.1016/j.gloplacha.2012.08.009.

4. Mu C, Abbott BW, Norris AJ, Mu M, Fan C, Chen X, et al. The status and stability of permafrost carbon on the Tibetan Plateau. Earth Sci Rev. 2020;211:103433. https://doi.org/10.1016/j.earscirev.2020.103433.

5. Rui JP, Li JB, Wang SP, An JX, Liu WT, Lin QY, et al. Responses of Bacterial Communities to Simulated Climate Changes in Alpine Meadow Soil of the Qinghai-Tibet Plateau. Appl Environ Microbiol. 2015;81(17):6070-7. https://doi.org/10.1128/aem.00557-15.

6. Cavicchioli R, Ripple WJ, Timmis KN, Azam F, Bakken LR, Baylis M, et al. Scientists' warning to humanity: microorganisms and climate change. Nat Rev Microbiol. 2019;17(9):569-86. https://doi.org/10.1038/s41579-019-0222-5.

7. Raymond PA, Hartmann J, Lauerwald R, Sobek S, McDonald C, Hoover M, et al. Global carbon dioxide emissions from inland waters. Nature. 2013;503(7476):355-9. 
https://doi.org/10.1038/nature12760.

8. DelSontro T, Beaulieu JJ, Downing JA. Greenhouse gas emissions from lakes and impoundments: Upscaling in the face of global change. Limnology Oceanography Letters. 2018;3(3):64-75. https://doi.org/10.1002/lol2.10073.

9. Saunois M, Bousquet P, Poulter B, Peregon A, Ciais P, Canadell JG, et al. The global methane budget 2000-2012. Earth System Science Data. 2016;8(2):697-751. https://doi.org/10.5194/essd-8-6972016.

10. Jousset A, Bienhold C, Chatzinotas A, Gallien L, Gobet A, Kurm V, et al. Where less may be more: how the rare biosphere pulls ecosystems strings. Isme Journal. 2017;11(4):853-62. https://doi.org/10.1038/ismej.2016.174.

11. Hu WG, Zhang Q, Tian T, Li DY, Cheng G, Mu J, et al. Characterization of the prokaryotic diversity through a stratigraphic permafrost core profile from the Qinghai-Tibet Plateau. Extremophiles. 2016;20(3):337-49. https://doi.org/10.1007/s00792-016-0825-y.

12. Hu WG, Zhang Q, Li DY, Cheng G, Mu J, Wu QB, et al. Diversity and community structure of fungi through a permafrost core profile from the Qinghai-Tibet Plateau of China. J Basic Microbiol. 2014;54(12):1331-41. https://doi.org/10.1002/jobm.201400232.

13. Wei SP, Cui HP, He H, Hu F, Su X, Zhu YH. Diversity and Distribution of Archaea Community along a Stratigraphic Permafrost Profile from Qinghai-Tibetan Plateau, China. Archaea-an International Microbiological Journal. 2014;12:240817. https://doi.org/10.1155/2014/240817.

14. Chen YL, Deng Y, Ding JZ, Hu HW, Xu TL, Li F, et al. Distinct microbial communities in the active and permafrost layers on the Tibetan Plateau. Mol Ecol. 2017;26(23):6608-20. https://doi.org/10.1111/mec.14396.

15. Han R, Zhang X, Liu J, Long QF, Chen LS, Liu DL, et al. Microbial community structure and diversity within hypersaline Keke Salt Lake environments. Can J Microbiol. 2017;63(11):895-908. https://doi.org/10.1139/cjm-2016-0773.

16. Xing R, Gao QB, Zhang FQ, Wang JL, Chen SL. Large-scale distribution of bacterial communities in the Qaidam Basin of the Qinghai-Tibet Plateau. Microbiologyopen. 2019;8(10):e909. https://doi.org/10.1002/mbo3.909.

17. Ma A, Zhang X, Jiang K, Zhao C, Liu J, Wu M, et al. Phylogenetic and Physiological Diversity of Cultivable Actinomycetes Isolated From Alpine Habitats on the Qinghai-Tibetan Plateau. Front Microbiol. 2020;11:555351. https://doi.org/10.3389/fmicb.2020.555351.

18. Ranjan R, Rani A, Metwally A, McGee HS, Perkins DL. Analysis of the microbiome: Advantages of whole genome shotgun versus $16 \mathrm{~S}$ amplicon sequencing. Biochem Biophys Res Commun. 2016;469(4):967-77. https://doi.org/10.1016/j.bbrc.2015.12.083.

19. Sunagawa S, Mende DR, Zeller G, Izquierdo-Carrasco F, Berger SA, Kultima JR, et al. Metagenomic species profiling using universal phylogenetic marker genes. Nat Methods. 2013;10(12):1196-9. https://doi.org/10.1038/nmeth.2693. 
20. Zhou JZ, He ZL, Yang YF, Deng Y, Tringe SG, Alvarez-Cohen L. High-Throughput Metagenomic Technologies for Complex Microbial Community Analysis: Open and Closed Formats. Mbio. 2015;6(1):e02288-14. https://doi.org/10.1128/mBio.02288-14.

21. Sharpton TJ. An introduction to the analysis of shotgun metagenomic data. Front Plant Sci. 2014;5:209. https://doi.org/10.3389/fpls.2014.00209.

22. Wiseschart A, Mhuantong W, Tangphatsornruang S, Chantasingh D, Pootanakit K. Shotgun metagenomic sequencing from Manao-Pee cave, Thailand, reveals insight into the microbial community structure and its metabolic potential. Bmc Microbiology. 2019;19:144. https://doi.org/10.1186/s12866-019-1521-8.

23. Alneberg J, Karlsson CMG, Divne A-M, Bergin C, Homa F, Lindh MV, et al. Genomes from uncultivated prokaryotes: a comparison of metagenome-assembled and single-amplified genomes. Microbiome. 2018;6:173. https://doi.org/10.1186/s40168-018-0550-0.

24. Sangwan N, Xia FF, Gilbert JA. Recovering complete and draft population genomes from metagenome datasets. Microbiome. 2016;4:8. https://doi.org/10.1186/s40168-016-0154-5.

25. Vanwonterghem I, Jensen PD, Rabaey K, Tyson GW. Genome-centric resolution of microbial diversity, metabolism and interactions in anaerobic digestion. Environ Microbiol. 2016;18(9):3144-58. https://doi.org/10.1111/1462-2920.13382.

26. Chen SF, Zhou YQ, Chen YR, Gu J. fastp: an ultra-fast all-in-one FASTQ preprocessor. Bioinformatics. 2018;34(17):884-90. https://doi.org/10.1093/bioinformatics/bty560.

27. Cock PJA, Fields CJ, Goto N, Heuer ML, Rice PM. The Sanger FASTQ file format for sequences with quality scores, and the Solexa/Illumina FASTQ variants. Nucleic Acids Res. 2010;38(6):1767-71. https://doi.org/10.1093/nar/gkp1137.

28. Uritskiy GV, DiRuggiero J, Taylor J. MetaWRAP-a flexible pipeline for genome-resolved metagenomic data analysis. Microbiome. 2018;6:158. https://doi.org/10.1186/s40168-018-0541-1.

29. Li D, Luo R, Liu C-M, Leung C-M, Ting H-F, Sadakane K, et al. MEGAHIT v1.0: A fast and scalable metagenome assembler driven by advanced methodologies and community practices. Methods. 2016;102:3-11. https://doi.org/10.1016/j.ymeth.2016.02.020.

30. Seemann T. Prokka: rapid prokaryotic genome annotation. Bioinformatics. 2014;30(14):2068-9. https://doi.org/10.1093/bioinformatics/btu153.

31. Patro R, Duggal G, Love MI, Irizarry RA, Kingsford C. Salmon provides fast and bias-aware quantification of transcript expression. Nat Methods. 2017;14(4):417-9. https://doi.org/10.1038/nmeth.4197.

32. Wood DE, Lu J, Langmead B. Improved metagenomic analysis with Kraken 2. Genome Biol. 2019;20(1):257. https://doi.org/10.1186/s13059-019-1891-0.

33. Lu J, Breitwieser FP, Thielen P, Salzberg SL. Bracken: estimating species abundance in metagenomics data. Peerj Computer Science. 2017:e104. https://doi.org/10.7717/peerj-cs.104.

34. Ondov BD, Bergman NH, Phillippy AM. Interactive metagenomic visualization in a Web browser. Bmc Bioinformatics. 2011;12:385. https://doi.org/10.1186/1471-2105-12-385. 
35. Buchfink B, Xie C, Huson DH. Fast and sensitive protein alignment using DIAMOND. Nat Methods. 2015;12(1):59-60. https://doi.org/10.1038/nmeth.3176.

36. Altschul SF, Gish W, Miller W, Myers EW, Lipman DJ. Basic local alignment search tool. Journal of molecular biology. 1990;215(3):403-10. https://doi.org/10.1006/jmbi.1990.9999.

37. Lombard V, Ramulu HG, Drula E, Coutinho PM, Henrissat B. The carbohydrate-active enzymes database (CAZy) in 2013. Nucleic Acids Res. 2014;42(D1):D490-5. https://doi.org/10.1093/nar/gkt1178.

38. Winnenburg R, Baldwin TK, Urban M, Rawlings C, Kohler J, Hammond-Kosack KE. PHI-base: a new database for pathogen host interactions. Nucleic Acids Res. 2006;34:D459-64. https://doi.org/10.1093/nar/gkj047.

39. Chen LH, Zheng DD, Liu B, Yang J, Jin QVFDB. 2016: hierarchical and refined dataset for big data analysis-10 years on. Nucleic Acids Research. 2016;44(D1):D694-D697. https://doi.org/10.1093/nar/gkv1239.

40. Pal C, Bengtsson-Palme J, Rensing C, Kristiansson E, Larsson DGJ. BacMet: antibacterial biocide and metal resistance genes database. Nucleic Acids Res. 2014;42(D1):D737-43. https://doi.org/10.1093/nar/gkt1252.

41. Alcock BP, Raphenya AR, Lau TTY, Tsang KK, Bouchard M, Edalatmand A, et al. CARD 2020: antibiotic resistome surveillance with the comprehensive antibiotic resistance database. Nucleic Acids Res. 2020;48(D1):D517-25. https://doi.org/10.1093/nar/gkz935.

42. Kanehisa M, Goto SKEGG. Kyoto Encyclopedia of Genes and Genomes. Nucleic Acids Res. 2000;28(1):27-30. https://doi.org/10.1093/nar/28.1.27.

43. Moriya Y, Itoh M, Okuda S, Yoshizawa AC, Kanehisa M. KAAS: an automatic genome annotation and pathway reconstruction server. Nucleic Acids Res. 2007;35:W182-5. https://doi.org/10.1093/nar/gkm321.

44. Tatusov RL, Koonin EV, Lipman DJ. A genomic perspective on protein families. Science. 1997;278(5338):631-7. https://doi.org/10.1126/science.278.5338.631.

45. Carbon S, Dietze H, Lewis SE, Mungall CJ, Munoz-Torres MC, Basu S, et al. Expansion of the Gene Ontology knowledgebase and resources. Nucleic Acids Res. 2017;45(D1):D331-8. https://doi.org/10.1093/nar/gkw1108.

46. Ashburner M, Ball CA, Blake JA, Botstein D, Butler H, Cherry JM, et al. Gene Ontology: tool for the unification of biology. Nat Genet. 2000;25(1):25-9. https://doi.org/10.1038/75556.

47. Carbon S, Douglass E, Dunn N, Good B, Harris NL, Lewis SE, et al. The Gene Ontology Resource: 20 years and still GOing strong. Nucleic Acids Res. 2019;47(D1):D330-8. https://doi.org/10.1093/nar/gky1055.

48. Huerta-Cepas J, Forslund K, Coelho LP, Szklarczyk D, Jensen LJ, von Mering C, et al. Fast GenomeWide Functional Annotation through Orthology Assignment by eggNOG-Mapper. Mol Biol Evol. 2017;34(8):2115-22. https://doi.org/10.1093/molbev/msx148. 
49. Huerta-Cepas J, Szklarczyk D, Heller D, Hernandez-Plaza A, Forslund SK, Cook H, et al. eggNOG 5.0: a hierarchical, functionally and phylogenetically annotated orthology resource based on 5090 organisms and 2502 viruses. Nucleic Acids Res. 2019;47(D1):D309-14. https://doi.org/10.1093/nar/gky1085.

50. Ye J, Zhang Y, Cui HH, Liu JW, Wu YQ, Cheng Y, et al. WEGO 2.0: a web tool for analyzing and plotting GO annotations, 2018 update. Nucleic Acids Res. 2018;46(W1):W71-5. https://doi.org/10.1093/nar/gky400.

51. Kang DWD, Li F, Kirton E, Thomas A, Egan R, An H, et al. MetaBAT 2: an adaptive binning algorithm for robust and efficient genome reconstruction from metagenome assemblies. Peerj. 2019;7:e7359. https://doi.org/10.7717/peerj.7359.

52. Wu YW, Simmons BA, Singer SW. MaxBin 2.0: an automated binning algorithm to recover genomes from multiple metagenomic datasets. Bioinformatics. 2016;32(4):605-7. https://doi.org/10.1093/bioinformatics/btv638.

53. Alneberg J, Bjarnason BS, de Bruijn I, Schirmer M, Quick J, ljaz UZ, et al. Binning metagenomic contigs by coverage and composition. Nat Methods. 2014;11(11):1144-6. https://doi.org/10.1038/nmeth.3103.

54. Parks DH, Imelfort M, Skennerton CT, Hugenholtz P, Tyson GW. CheckM: assessing the quality of microbial genomes recovered from isolates, single cells, and metagenomes. Genome Res. 2015;25(7):1043-55. https://doi.org/10.1101/gr.186072.114.

55. Momper L, Jungbluth SP, Lee MD, Amend JP. Energy and carbon metabolisms in a deep terrestrial subsurface fluid microbial community. Isme Journal. 2017;11(10):2319-33. https://doi.org/10.1038/ismej.2017.94.

56. Creevey CJ, Doerks T, Fitzpatrick DA, Raes J, Bork P. Universally Distributed Single-Copy Genes Indicate a Constant Rate of Horizontal Transfer. Plos One. 2011;6(8):e22099. https://doi.org/10.1371/journal.pone.0022099.

57. Chaumeil P-A, Mussig AJ, Hugenholtz P, Parks DH. GTDB-Tk: a toolkit to classify genomes with the Genome Taxonomy Database. Bioinformatics. 2020;36(6):1925-7. https://doi.org/10.1093/bioinformatics/btz848.

58. Parks DH, Chuvochina M, Chaumeil P-A, Rinke C, Mussig AJ, Hugenholtz P. A complete domain-tospecies taxonomy for Bacteria and Archaea. Nat Biotechnol. 2020;38(9):1079-86. https://doi.org/10.1038/s41587-020-0501-8.

59. Parks DH, Chuvochina M, Waite DW, Rinke C, Skarshewski A, Chaumeil P-A, et al. A standardized bacterial taxonomy based on genome phylogeny substantially revises the tree of life. Nat Biotechnol. 2018;36(10):996-1004. https://doi.org/10.1038/nbt.4229.

60. Price MN, Dehal PS, Arkin AP. FastTree 2-Approximately Maximum-Likelihood Trees for Large Alignments. Plos One. 2010;5(3):e9490. https://doi.org/10.1371/journal.pone.0009490.

61. Letunic I, Bork P. Interactive Tree Of Life (iTOL) v4: recent updates and new developments. Nucleic Acids Res. 2019;47(W1):W256-9. https://doi.org/10.1093/nar/gkz239. 
62. Yin YB, Mao XZ, Yang JC, Chen X, Mao FL, Xu Y. dbCAN: a web resource for automated carbohydrateactive enzyme annotation. Nucleic Acids Res. 2012;40(W1):W445-51. https://doi.org/10.1093/nar/gks479.

63. Lannes R, Olsson-Francis K, Lopez P, Bapteste E. Carbon Fixation by Marine Ultrasmall Prokaryotes. Genome Biology Evolution. 2019;11(4):1166-77. https://doi.org/10.1093/gbe/evz050.

64. Aziz RK, Bartels D, Best AA, DeJongh M, Disz T, Edwards RA, et al. The RAST server: Rapid annotations using subsystems technology. Bmc Genomics. 2008;9:75. https://doi.org/10.1186/1471-2164-9-75.

65. Overbeek R, Olson R, Pusch GD, Olsen GJ, Davis JJ, Disz T, et al. The SEED and the Rapid Annotation of microbial genomes using Subsystems Technology (RAST). Nucleic Acids Res. 2014;42(D1):D206-14. https://doi.org/10.1093/nar/gkt1226.

66. Brettin T, Davis JJ, Disz T, Edwards RA, Gerdes S, Olsen GJ, et al. RASTtk: A modular and extensible implementation of the RAST algorithm for building custom annotation pipelines and annotating batches of genomes. Sci Rep. 2015;5:8365. https://doi.org/10.1038/srep08365.

67. Blin K, Shaw S, Steinke K, Villebro R, Ziemert N, Lee SY, et al. antiSMASH 5.0: updates to the secondary metabolite genome mining pipeline. Nucleic Acids Res. 2019;47(W1):W81-7. https://doi.org/10.1093/nar/gkz310.

68. Edgar RC. MUSCLE: multiple sequence alignment with high accuracy and high throughput. Nucleic Acids Res. 2004;32(5):1792-7. https://doi.org/10.1093/nar/gkh340.

69. Keto-Timonen R, Hietala N, Palonen E, Hakakorpi A, Lindstrom M, Korkeala H. Cold Shock Proteins: A Minireview with Special Emphasis on Csp-family of Enteropathogenic Yersinia. Front Microbiol. 2016;7:1151. https://doi.org/10.3389/fmicb.2016.01151.

70. Drewniak L, Krawczyk PS, Mielnicki S, Adamska D, Sobczak A, Lipinski L, et al. Physiological and Metagenomic Analyses of Microbial Mats Involved in Self-Purification of Mine Waters Contaminated with Heavy Metals. Front Microbiol. 2016;7:1252. https://doi.org/10.3389/fmicb.2016.01252.

71. Huang Q, Briggs BR, Dong H, Jiang H, Wu G, Edwardson C, et al. Taxonomic and Functional Diversity Provides Insight into Microbial Pathways and Stress Responses in the Saline Qinghai Lake, China. Plos One. 2014;9(11):e111681. https://doi.org/10.1371/journal.pone.0111681.

72. Wang R, Han R, Long Q, Gao X, Xing J, Shen G, et al. Bacterial and Archaeal Communities within an Ultraoligotrophic, High-altitude Lake in the Pre-Himalayas of the Qinghai-Tibet Plateau. Indian Journal of Microbiology. 2020;60(3):363-73. https://doi.org/10.1007/s12088-020-00881-8.

73. Naghoni A, Emtiazi G, Amoozegar MA, Cretoiu MS, Stal LJ, Etemadifar Z, et al. Microbial diversity in the hypersaline Lake Meyghan, Iran. Sci Rep. 2017;7:11522. https://doi.org/10.1038/s41598-01711585-3.

74. Genilloud O. Actinomycetes: still a source of novel antibiotics. Nat Prod Rep. 2017;34(10):1203-32. https://doi.org/10.1039/c7np00026j.

75. Ventola CL. The antibiotic resistance crisis: part 1: causes and threats. P \& T: a peer-reviewed journal for formulary management. 2015;40(4):277 - 83. 
76. Gaete A, Mandakovic D, Gonzalez M. Isolation and Identification of Soil Bacteria from Extreme Environments of Chile and Their Plant Beneficial Characteristics. Microorganisms. 2020;8(8):1213. https://doi.org/10.3390/microorganisms8081213.

77. Zhang J, Sun QL, Zeng ZG, Chen S, Sun L. Microbial diversity in the deep-sea sediments of Iheya North and Iheya Ridge, Okinawa Trough. Microbiol Res. 2015;177:43-52. https://doi.org/10.1016/j.micres.2015.05.006.

78. Chakraborty J, Sapkale V, Rajput V, Shah M, Kamble S, Dharne M. Shotgun metagenome guided exploration of anthropogenically driven resistomic hotspots within Lonar soda lake of India. Ecotoxicol Environ Saf. 2020;194:110443. https://doi.org/10.1016/j.ecoenv.2020.110443.

79. Philippe N, Legendre M, Doutre G, Coute Y, Poirot O, Lescot M, et al. Pandoraviruses: Amoeba Viruses with Genomes Up to $2.5 \mathrm{Mb}$ Reaching That of Parasitic Eukaryotes. Science. 2013;341(6143):281-6. https://doi.org/10.1126/science.1239181.

80. La Cono V, Ruggeri G, Azzaro M, Crisafi F, Decembrini F, Denaro R, et al. Contribution of Bicarbonate Assimilation to Carbon Pool Dynamics in the Deep Mediterranean Sea and Cultivation of Actively Nitrifying and CO2-Fixing Bathypelagic Prokaryotic Consortia. Front Microbiol. 2018;9:3. https://doi.org/10.3389/fmicb.2018.00003.

81. Berg IA. Ecological Aspects of the Distribution of Different Autotrophic CO2 Fixation Pathways. Appl Environ Microbiol. 2011;77(6):1925-36. https://doi.org/10.1128/aem.02473-10.

82. Zhang X, Xu W, Liu Y, Cai M, Luo Z, Li M. Metagenomics Reveals Microbial Diversity and Metabolic Potentials of Seawater and Surface Sediment From a Hadal Biosphere at the Yap Trench. Front Microbiol. 2018;9:2402. https://doi.org/10.3389/fmicb.2018.02402.

83. Magnabosco C, Ryan K, Lau MCY, Kuloyo O, Lollar BS, Kieft TL, et al. A metagenomic window into carbon metabolism at $3 \mathrm{~km}$ depth in Precambrian continental crust. Isme Journal. 2016;10(3):73041. https://doi.org/10.1038/ismej.2015.150.

84. Ruiz-Fernandez P, Ramirez-Flandes S, Rodriguez-Leon E, Ulloa O. Autotrophic carbon fixation pathways along the redox gradient in oxygen-depleted oceanic waters. Environmental Microbiology Reports. 2020;12(3):334-41. https://doi.org/10.1111/1758-2229.12837.

85. Cai Y, Zheng Y, Bodelier PLE, Conrad R, Jia Z. Conventional methanotrophs are responsible for atmospheric methane oxidation in paddy soils. Nat Commun. 2016;7:11728. https://doi.org/10.1038/ncomms11728.

86. Nakicenovic N, Alcamo J, Grubler A, Riahi K, Roehrl RA, Rogner H-H, et al. Special Report on Emissions Scenarios, Working Group III, Intergovernmental Panel on Climate Change. Cambridge University Press; 2000.

87. Rathour R, Gupta J, Mishra A, Rajeev AC, Dupont CL, Thakur IS. A comparative metagenomic study reveals microbial diversity and their role in the biogeochemical cycling of Pangong lake. Sci Total Environ. 2020;731:139074. https://doi.org/10.1016/j.scitotenv.2020.139074.

88. Canelhas MR, Denfeld BA, Weyhenmeyer GA, Bastviken D, Bertilsson S. Methane oxidation at the water-ice interface of an ice-covered lake. Limnol Oceanogr. 2016;61:78-90. 
https://doi.org/10.1002/Ino.10288.

89. Wang SY, Liu WY, Zhao SY, Wang C, Zhuang LJ, Liu L, et al. Denitrification is the main microbial N loss pathway on the Qinghai-Tibet Plateau above an elevation of $5000 \mathrm{~m}$. Sci Total Environ. 2019;696:133852. https://doi.org/10.1016/j.scitotenv.2019.133852.

90. Spasov E, Tsuji JM, Hug LA, Doxey AC, Sauder LA, Parker WJ, et al. High functional diversity among Nitrospira populations that dominate rotating biological contactor microbial communities in a municipal wastewater treatment plant. Isme Journal. 2020;14(7):1857-72. https://doi.org/10.1038/s41396-020-0650-2.

91. Rasigraf O, Schmitt J, Jetten MSM, Luke C. Metagenomic potential for and diversity of N-cycle driving microorganisms in the Bothnian Sea sediment. Microbiologyopen. 2017;6(4):e475. https://doi.org/10.1002/mbo3.475.

92. Ren $M$, Zhang Z, Wang X, Zhou Z, Chen D, Zeng H, et al. Diversity and Contributions to Nitrogen Cycling and Carbon Fixation of Soil Salinity Shaped Microbial Communitiesin Tarim Basin. Front Microbiol. 2018;9:431. https://doi.org/10.3389/fmicb.2018.00431.

93. Cao H, Wang Y, Lee OO, Zeng X, Shao Z, Qian P-Y. Microbial Sulfur Cycle in Two Hydrothermal Chimneys on the Southwest Indian Ridge. Mbio. 2014;5(1):e00980-13. https://doi.org/10.1128/mBio.00980-13.

94. Neumann S, Wynen A, Truper HG, Dahl C. Characterization of the cys gene locus from Allochromatium vinosum indicates an unusual sulfate assimilation pathway. Mol Biol Rep. 2000;27(1):27-33. https://doi.org/10.1023/a:1007058421714.

95. Koo H, Hakim JA, Morrow CD, Crowley MR, Andersen DT, Bej AK. Metagenomic Analysis of Microbial Community Compositions and Cold-Responsive Stress Genes in Selected Antarctic Lacustrine and Soil Ecosystems. Life-Basel. 2018;8(3):29. https://doi.org/10.3390/life8030029.

96. Song W, Qi R, Zhao L, Xue N, Wang L, Yang Y. Bacterial community rather than metals shaping metal resistance genes in water, sediment and biofilm in lakes from arid northwestern China. Environ Pollut. 2019;254:113041. https://doi.org/10.1016/j.envpol.2019.113041.

97. Wu J, Lu J, Li LM, Min XY, Luo YM. Pollution, ecological-health risks, and sources of heavy metals in soil of the northeastern Qinghai-Tibet Plateau. Chemosphere. 2018;201:234-42. https://doi.org/10.1016/j.chemosphere.2018.02.122.

98. Chen Y, Jiang YM, Huang HY, Mou LC, Ru JL, Zhao JH, et al. Long-term and high-concentration heavy-metal contamination strongly influences the microbiome and functional genes in Yellow River sediments. Sci Total Environ. 2018;637:1400-12. https://doi.org/10.1016/j.scitotenv.2018.05.109.

99. Yamada Y, Kuzuyama T, Komatsu M, Shin-ya K, Omura S, Cane DE, et al. Terpene synthases are widely distributed in bacteria. Proc Natl Acad Sci USA. 2015;112(3):857-62. https://doi.org/10.1073/pnas.1422108112.

100. Cuadrat RRC, lonescu D, Davila AMR, Grossart H-P. Recovering Genomics Clusters of Secondary Metabolites from Lakes Using Genome-Resolved Metagenomics. Front Microbiol. 2018;9:251. https://doi.org/10.3389/fmicb.2018.00251. 
101. Finking R, Marahiel MA. Biosynthesis of nonribosomal peptides. Annu Rev Microbiol. 2004;58:45388. https://doi.org/10.1146/annurev.micro.58.030603.123615.

102. Ma Y, Wilson CA, Novak JT, Riffat R, Aynur S, Murthy S, et al. Effect of Various Sludge Digestion Conditions on Sulfonamide, Macrolide, and Tetracycline Resistance Genes and Class I Integrons. Environmental Science Technology. 2011;45(18):7855-61. https://doi.org/10.1021/es200827t.

103. Pan X, Lin L, Zhang WH, Dong L, Yang YY. Metagenome sequencing to unveil the resistome in a deep subtropical lake on the Yunnan-Guizhou Plateau, China. Environ Pollut. 2020;263:114470. https://doi.org/10.1016/j.envpol.2020.114470.

104. Jiang XL, Ellabaan MMH, Charusanti P, Munck C, Blin K, Tong YJ, et al. Dissemination of antibiotic resistance genes from antibiotic producers to pathogens. Nat Commun. 2017;8:15784. https://doi.org/10.1038/ncomms15784.

\section{Tables}

Table 1 Summary of the sample information and the metagenomic sequencing results

\begin{tabular}{ccc}
\hline & $s 05-02$ (soil) & s05-03 (water) \\
\hline Longitude & 31.34 & 31.34 \\
Latitude & 90.60 & 00.60 \\
Raw reads & $196,179,536$ & $160,824,924$ \\
Raw bases (bp) & $29,623,109,936$ & $24,284,563,524$ \\
Clean reads (\%) & $194,202,138(98.99 \%)$ & $159,527,496(99.19 \%)$ \\
Clean bases (\%) & $29,290,018,832(98.88 \%)$ & $24,062,066,782(99.08 \%)$ \\
Contigs Num & 160,212 & 135,994 \\
Total length (bp) & $356,933,003$ & $397,377,554$ \\
Max length (bp) & 833,193 & 286,311 \\
N50 (bp) & 2,289 & 3,841 \\
GC content (\%) & 60.18 & 51.33 \\
Average coverage (\%) & 31.45 & 45.70 \\
Percent of reference bases covered (\%) & 99.90 & 99.92 \\
\hline
\end{tabular}

Table 2 Summary of functional gene annotations against different databases in the two samples 


\begin{tabular}{ccc}
\hline Database & s05-02 (soil) & s05-03 (water) \\
\hline Predicted genes & 351,242 & 390,943 \\
nr & 232,679 & 294,919 \\
KEGG & 104,704 & 117,467 \\
COG & 211,757 & 222,635 \\
GO & 45,638 & 59,921 \\
CAZy & 52,427 & 51,940 \\
VFDB & 45,976 & 45,547 \\
BacMet & 21,273 & 18,305 \\
PHI & 59,516 & 60,782 \\
CARD & 11 & 12 \\
\hline
\end{tabular}

\section{Figures}

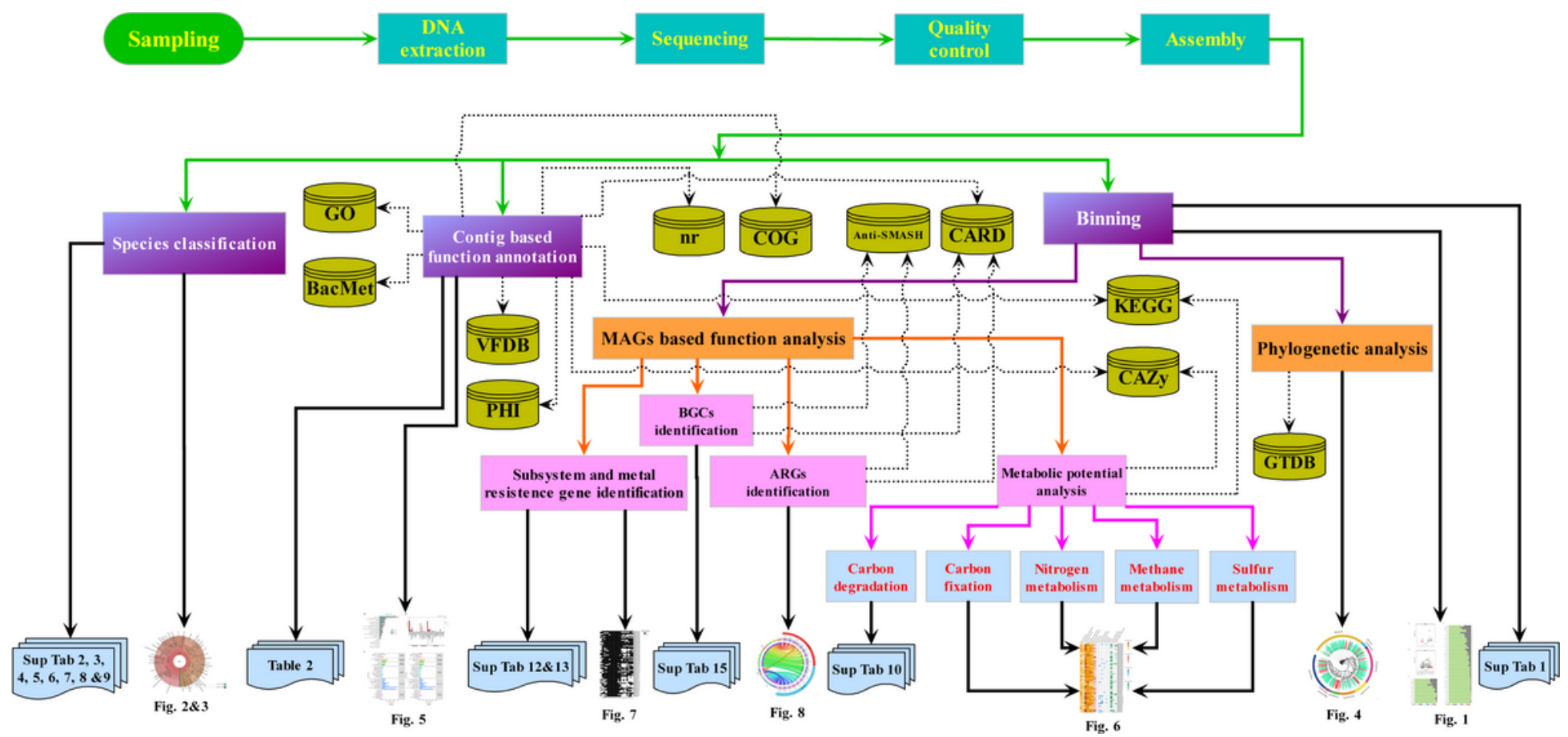

Figure 1

Analysis flow chart in this study. MAGs: metagenome-assembled genomes; BGC: Biosynthetic gene cluster; ARG: Antibiotics resistance gene. Sup Tab 1 denotes Table S3, Sup Tab 2 denotes Table S4, etc. The detailed information of the 11 databases in the figure can be referred to the reference list. 
a

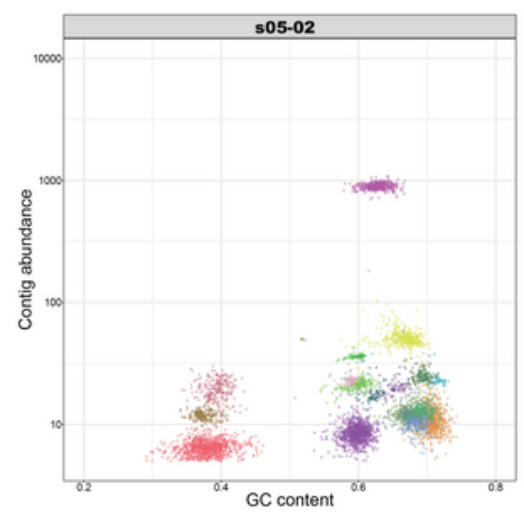

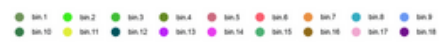

b
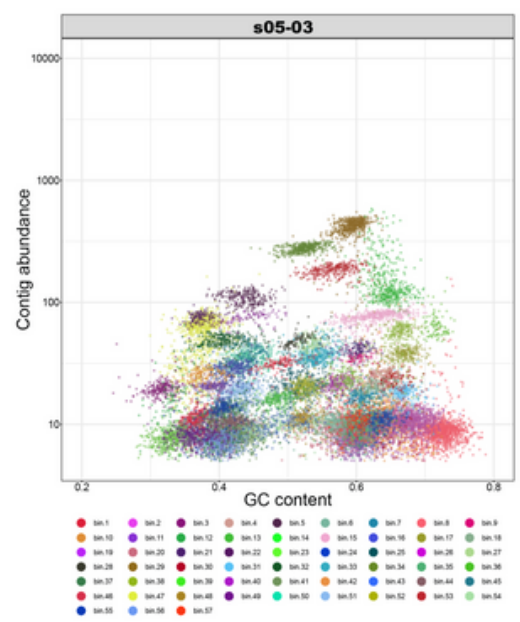

c

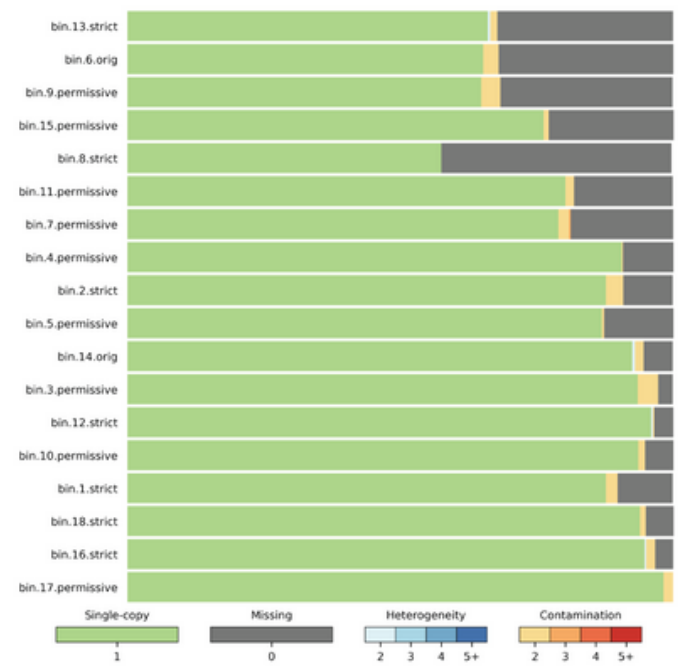

d

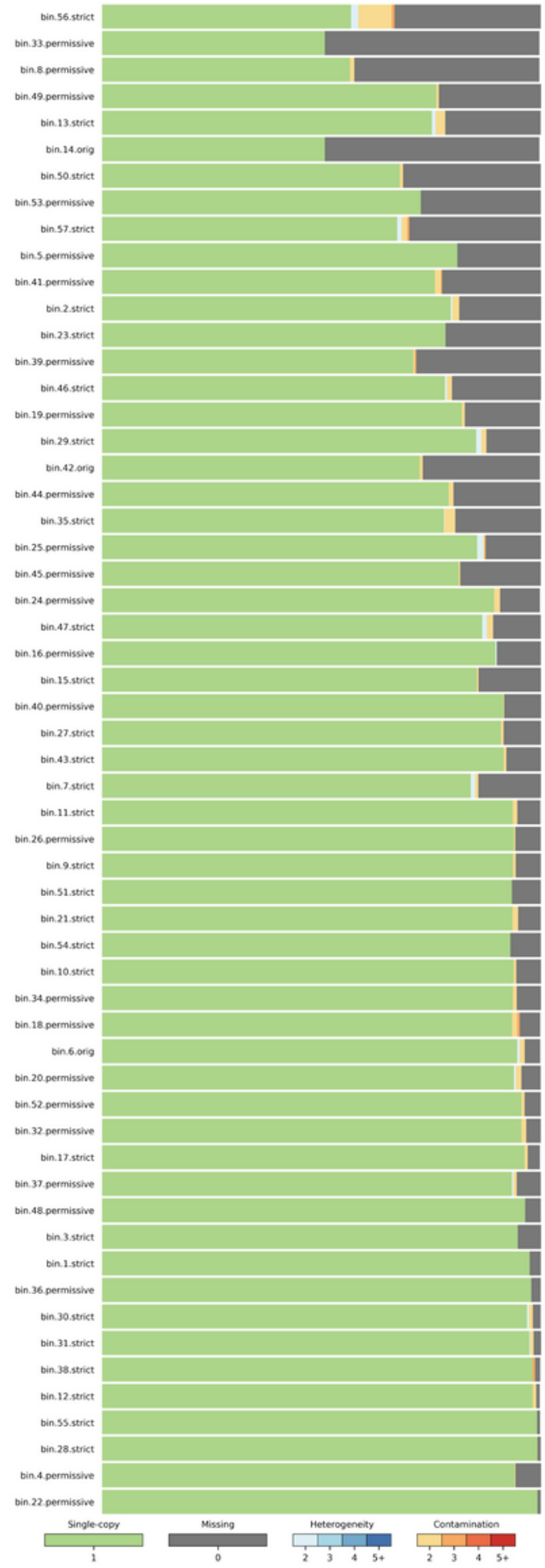

Figure 2

The evaluations of binning and reassembled MAGs. a (s05-02, soil), and b (s05-03, water): The distribution of the contigs in each bin after refining. The abscissa represents the GC content of the contigs, and the ordinate represents the contig abundance, with one dot representing one contig. The contigs in same color belong to the same bin. c (s05-02) and d (s05-03): An evaluation of the bins after 
reassembling using the reassemble_bins module in MetaWRAP [28]. The figures indicate Single-copy, Missing, Heterogeneity and Contamination of the reassembled bins.

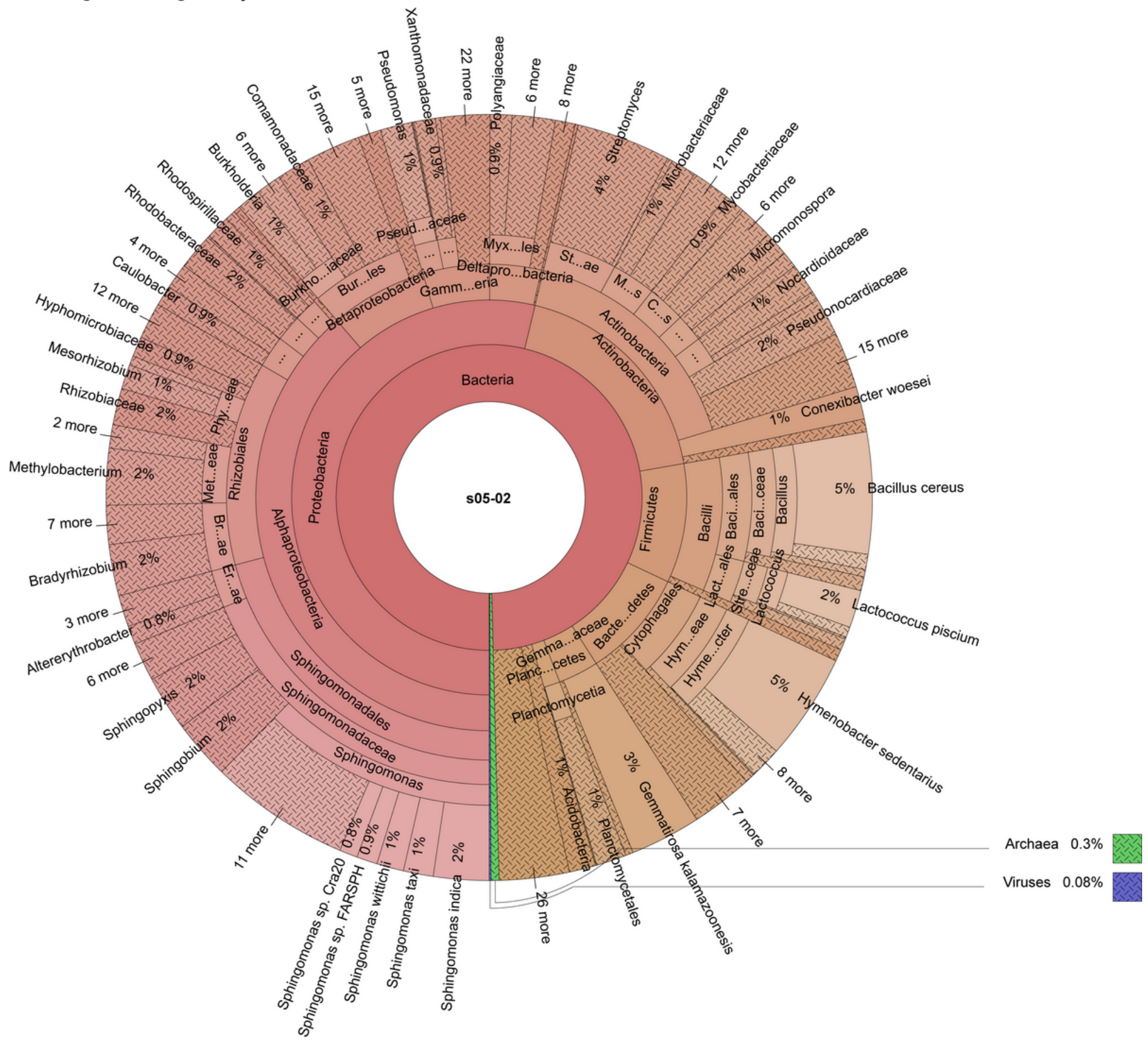

\section{Figure 3}

The composition of the soil microbial community. The figure shows the relatively complete distribution at species level. 


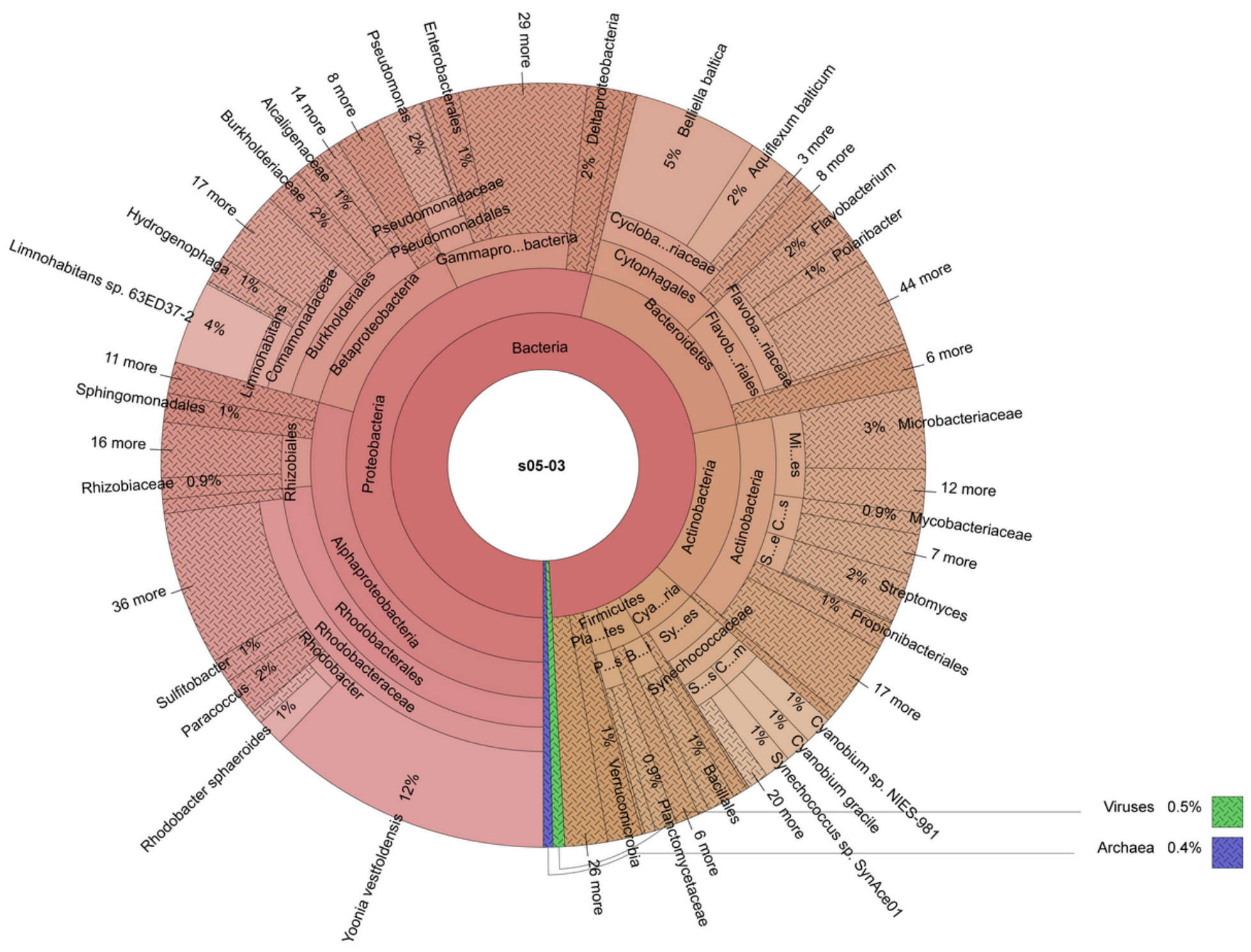

Figure 4

The overall composition of the microorganisms in the water. The figure shows the most abundant taxa, with the remaining categories to be omitted. 


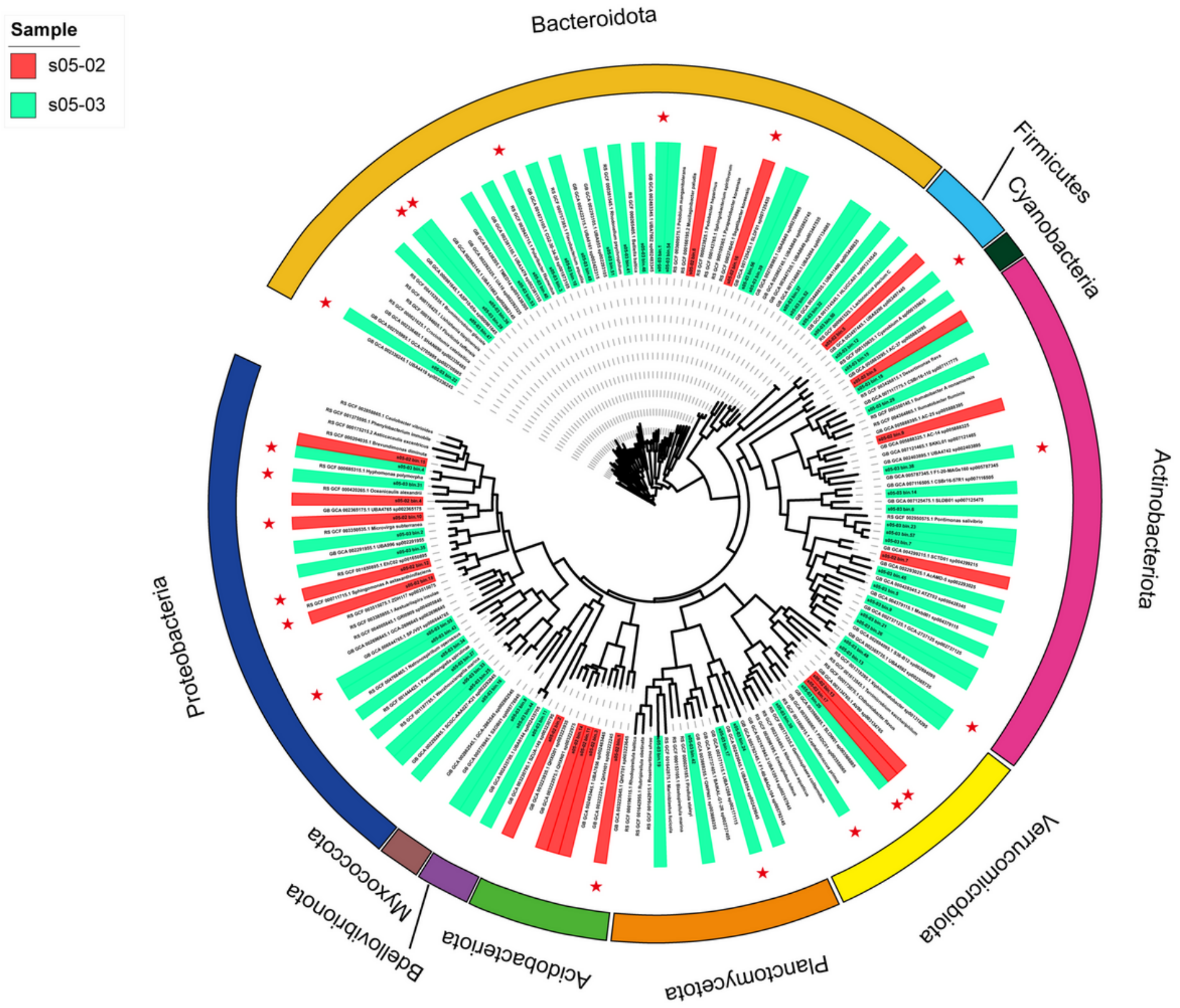

\section{Figure 5}

Phylogenetic tree of 75 reconstructed metagenome-assembled genomes (MAGs). The maximum likelihood evolutionary tree is reconstructed for all the $75 \mathrm{MAGs}$ and their nearest neighbor reference genomes. The MAGs of s05-02 were represented by the red background, and the MAGs of s05-03 by the green background. High quality MAGs, namely MAGs with completeness $>95 \%$ and contamination $<5 \%$, are marked with a star sign, and the phyla to which they belong were distinguished in outer rings in different colors. 

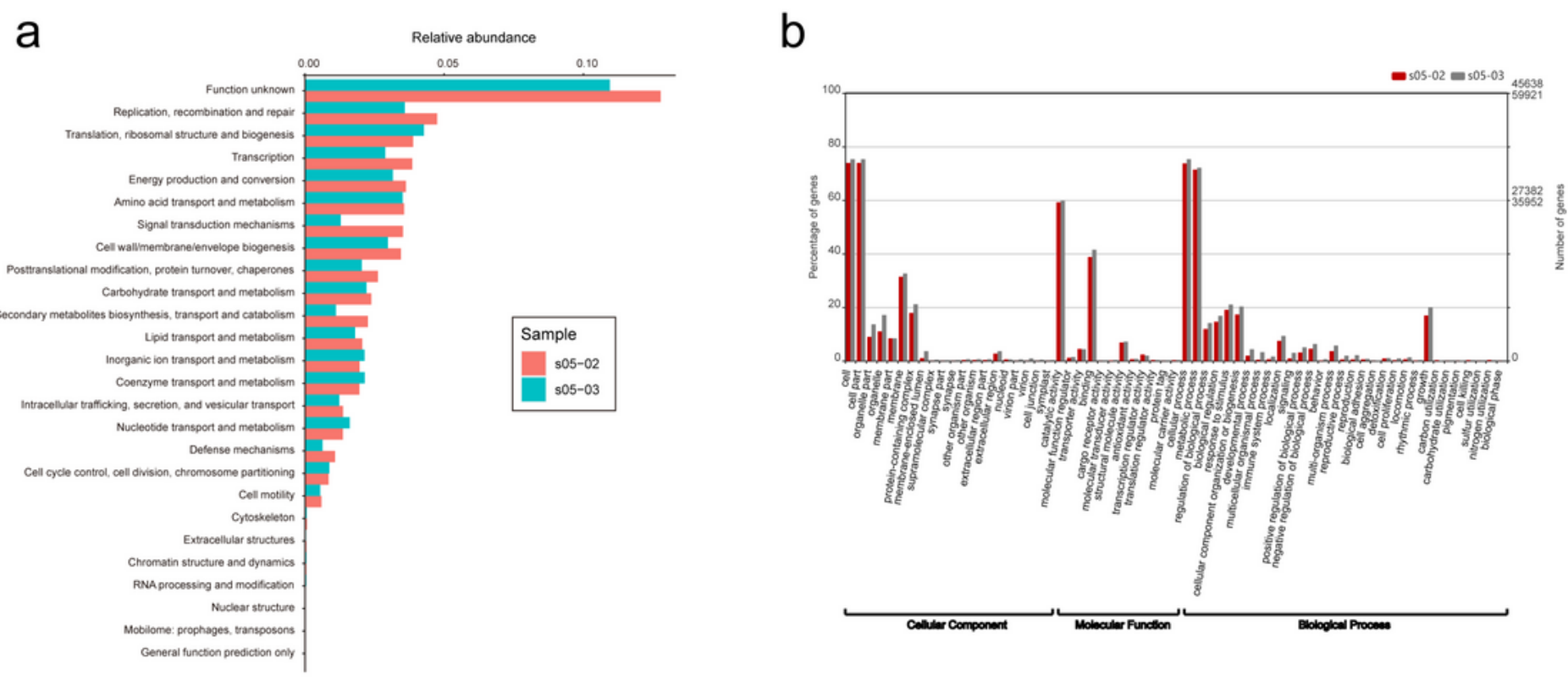

C
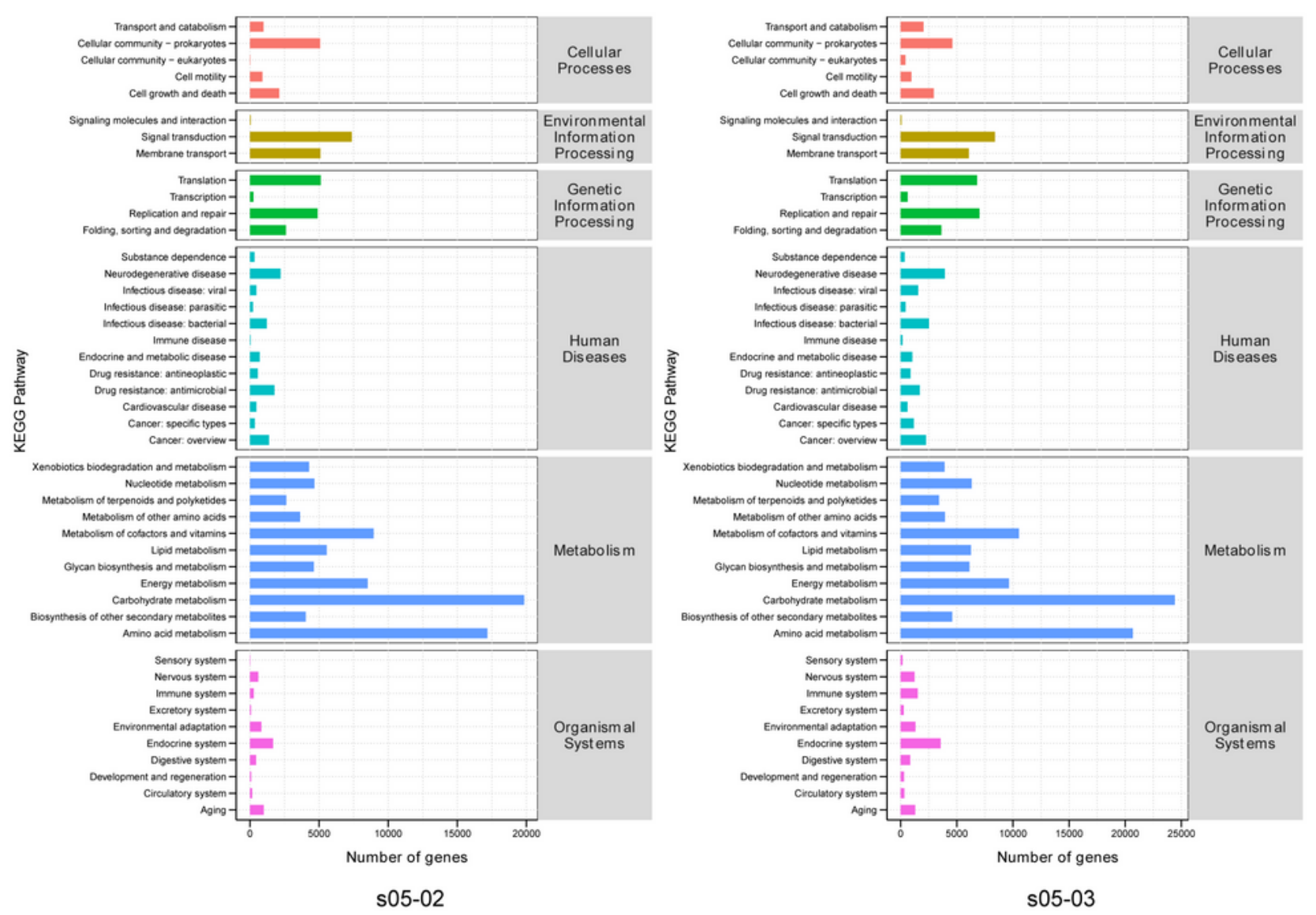

\section{Figure 6}

Visualization of functional genes annotation (a) The relative abundance of the COG [44] functional categories of the two samples. The red bars represent s05-02 (soil), and the green bars represent s05-03 (water). (b) GO distribution analysis of the two samples. The horizontal axis indicates the GO [46] functional classifications, with the red bars representing s05-02, and the grey bars representing s05-03. All the 2-level GO terms are arranged in three main categories (cellular component, molecular function 
and biological process). (c) The number of the genes annotated at the first two levels KEGG [42] pathway. S05-02 is displayed on the left and s05-03 on the right. The horizontal axes represent the number of the genes, and the vertical axes represent the metabolic pathways.

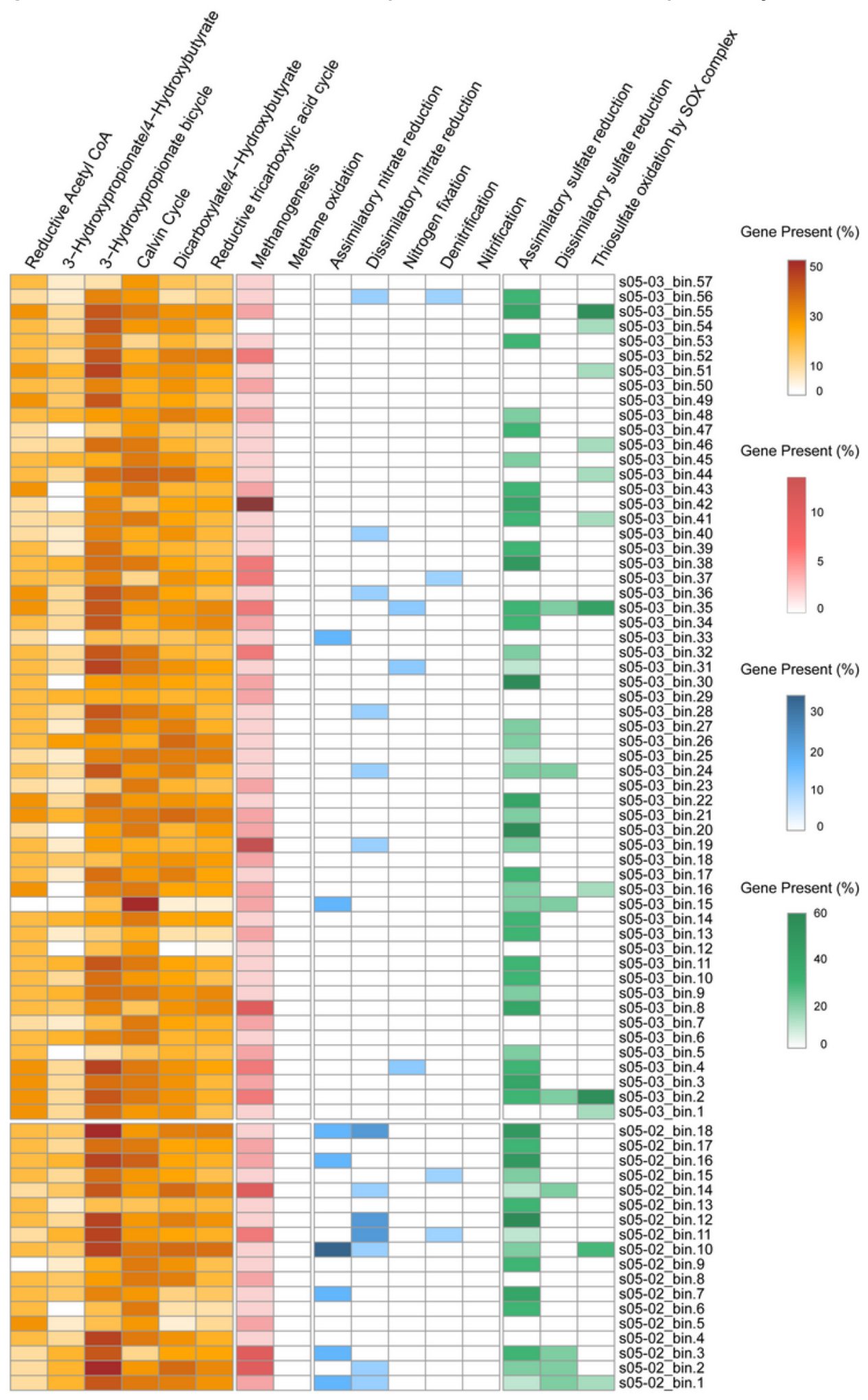

Figure 7

KEGG [42] annotation of energy metabolism for the two samples. The heatmap shows the percentage of the associated genes. The orange boxes represent carbon fixation; the red boxes represent methane 
metabolism; the blue boxes represent nitrogen metabolism; the green boxes represent sulfur metabolism. Here, we investigated six carbon fixation pathways, the methanogenesis and methane oxidation pathways, nitrogen metabolism pathways, and sulfur metabolism pathways.

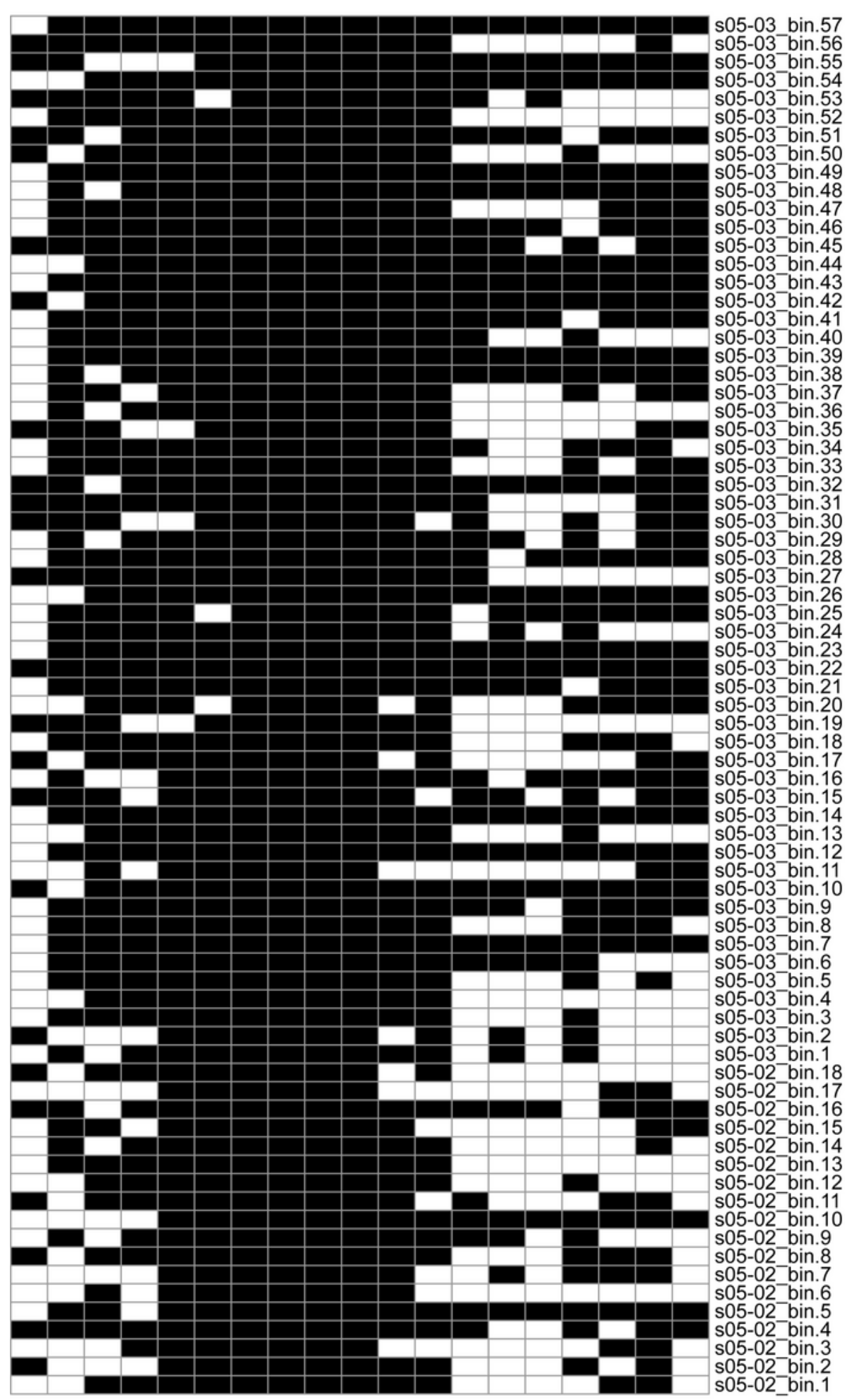

\section{Gene Presence \\ YES \\ NO}

V Mg Hg Te Se $\mathrm{Ni} \mathrm{Sb}$ As Mo W Co $\mathrm{Cr} \mathrm{Pb} \mathrm{Mn} \mathrm{Cd} \mathrm{Fe} \mathrm{Ag} \mathrm{Cu} \mathrm{Zn}$

\section{Figure 8}

Heavy metal resistance genes identified in the two samples. The black squares represent the presence of the related genes, and the blank squares represent the absence of the related genes. V: the resistant 
genes for vanadium; Mg: the resistant genes for magnesium; Hg: the resistant genes for mercury; Te: the resistant genes for tellurium; Se: the resistant genes for selenium; Ni: the resistant genes for nickel; Sb: the resistant genes for antimony; As: the resistant genes for arsenic; Mo: the resistant genes for molybdenum; W: the resistant genes for wolfram; Co: the resistant genes for cobalt; Cr: the resistant genes for chromium; $\mathrm{Pb}$ : the resistant genes for lead; $\mathrm{Mn}$ : the resistant genes for manganese; $\mathrm{Cd}$ : the resistant genes for cadmium; Fe: the resistant genes for iron; Ag: the resistant genes for silver; $\mathrm{Cu}$ : the resistant genes for copper; $\mathrm{Zn}$ : the resistant genes for zinc.

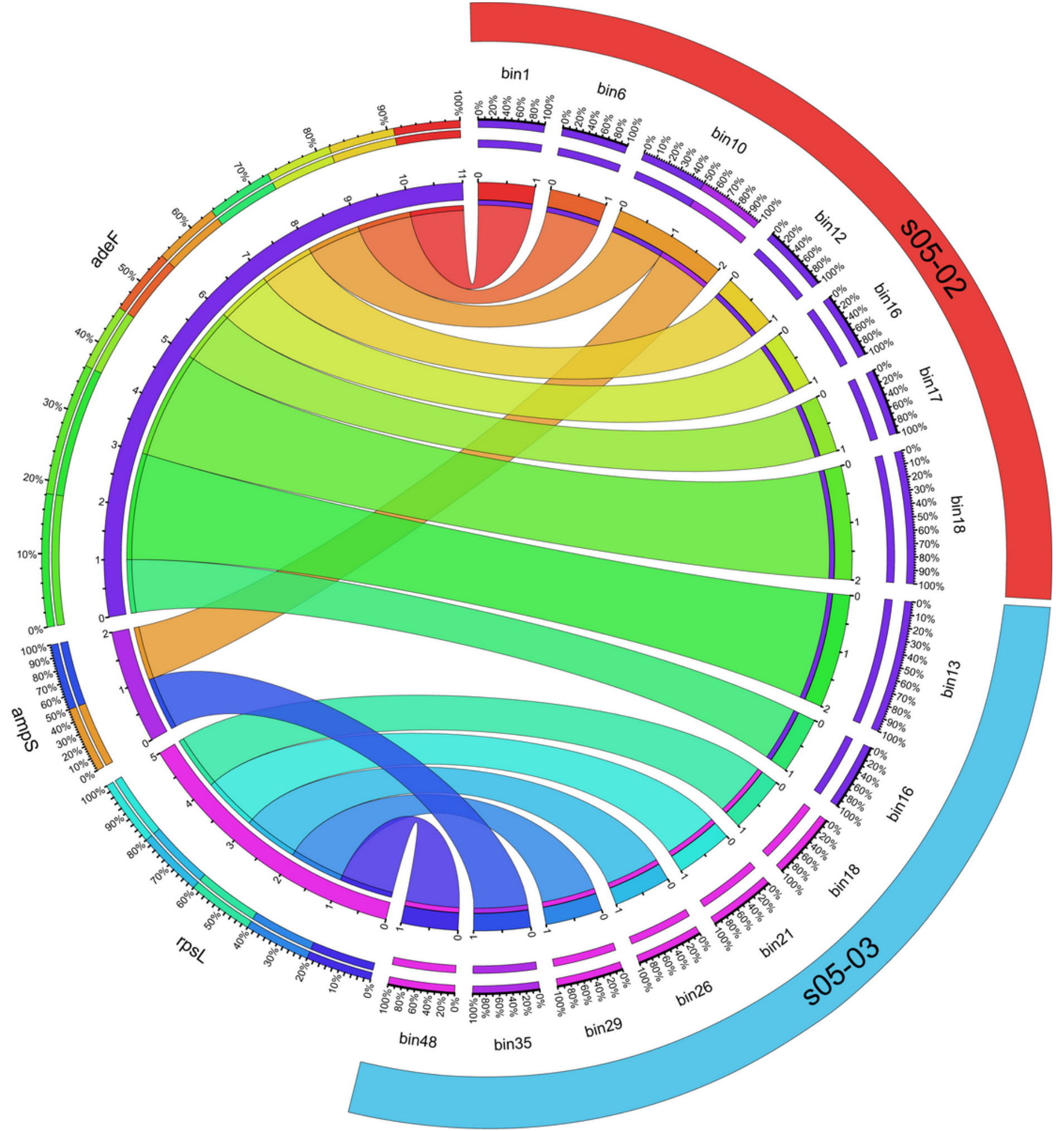




\section{Figure 9}

The circos of the annotation of MAGs by using CARD [41]. The ARGs information is on the left side of the peripheral ring, and the MAGs information is on the right side. The inner ring shows different MAGs and ARGs by different colors, and the scale represents the abundance information. The width of the band is proportional to the abundance of a particular ARG in each MAG.

\section{Supplementary Files}

This is a list of supplementary files associated with this preprint. Click to download.

- Tables1.pdf

- Tables10.pdf

- Tables11.pdf

- Tables12.pdf

- Tables13.pdf

- Tables14.pdf

- Tables15.pdf

- TableS16.pdf

- Tables2.pdf

- Tables3.pdf

- Tables4.pdf

- Tables5.pdf

- TableS6.pdf

- TableS7.pdf

- Tables8.pdf

- Tables9.pdf 